\title{
A Calderón Multiplicative Preconditioner for the Electromagnetic Poincaré-Steklov Operator of a Heterogeneous Domain with Scattering Applications
}

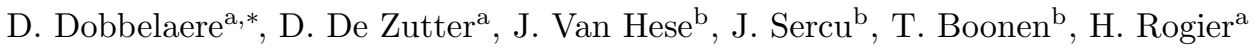 \\ ${ }^{a}$ Electromagnetics Group, Department of Information Technology, Ghent University, Sint-Pietersnieuwstraat 41, 9000 \\ Ghent, Belgium \\ ${ }^{b}$ Keysight Technologies Belgium N.V., Kortrijksesteenweg 1093B, 9051 Sint-Denijs-Westrem, Belgium
}

\begin{abstract}
In the context of hybrid formulations, the Poincaré-Steklov operator acting on traces of solutions to the vector Helmholtz equation in a heterogeneous interior domain with a smooth boundary is regularized by a well-known boundary integral operator related to the homogeneous exterior domain. For the first time, this property allows us to simultaneously construct a Calderón multiplicative preconditioner for the discretized operator and for a 3-D hybrid finite/boundary element method formulation, applicable to electromagnetic scattering problems. Numerical examples demonstrate the effectiveness of this novel preconditioning scheme, even for heterogeneous domains with non-smooth boundaries.
\end{abstract}

Keywords: Poincaré-Steklov operator, Heterogeneous domain, Calderón preconditioner, Schur complement discretization, Preconditioned hybrid formulation, Electromagnetic scattering

\section{Introduction}

A Poincaré-Steklov operator (PS for short), also called Dirichlet-to-Neumann operator, embodies a connection between the boundary values and (a function of) their derivatives of solutions to a boundary value problem of a linear partial differential operator. PS operators and their discretizations are present in continuous and discrete formulations of a wide spectrum of equations in computational physics, including heat problems, fluid dynamics [1], structural mechanics, acoustics and electromagnetics [2 5]. They naturally allow to impose field continuity constraints at the interface between physical domains and are consequently found in domain decomposition methods of finite element methods [6 8 and in hybrid finite/boundary element method (FEM-BEM) formulations 2, 3, 9. For differential operators acting on scalar fields, for example the Helmholtz operator $\Delta+k^{2}$, the PS operator maps the scalar boundary value of a solution onto its normal derivative [10. In this contribution, we focus on the vectorial time-harmonic electromagnetic PS operator mapping the tangential boundary value of the electric field $\mathbf{e}$, which satisfies the heterogeneous vector Helmholtz equation $\nabla \times \nabla \times \mathbf{e}+k^{2}(x, y, z) \mathbf{e}=0$ in the interior domain, onto the corresponding tangential component of the magnetic field trace [4, 5].

The presence of ill-conditioned discretized PS operators in numerical formulations severely increases the number of iterations and hampers the convergence speed of Krylov iterative methods. This has incited the development of preconditioners in past research, of which we intend to give a few examples. The NeumannNeumann and Interface Strip preconditioner have been proposed for the global PS operator in domain decomposition methods 1, 11. Moreover, we note that the application of a Calderón preconditioner for

\footnotetext{
* Corresponding author

Email addresses: dieter.dobbelaere@intec.ugent.be (D. Dobbelaere), daniel.dezutter@intec.ugent.be (D. De Zutter), hendrik.rogier@intec.ugent.be (H. Rogier)
} 
the Poincaré-Steklov operator of the 2-D scalar heterogeneous Helmholtz equation on a structured square domain has been investigated in [12].

In this contribution, we propose a Calderón multiplicative preconditioner for the finite element Schur complement discretization of the PS operator, denoted $\mathcal{P}$, for the vector heterogeneous Helmholtz equation on a general 3-D domain. First, we prove a decomposition of $\mathcal{P}$ in terms of well-known boundary integral operators. Then, we show that $\mathcal{P}$ is regularized by the so-called electric field integral operator $\mathcal{T}_{k}$, further defined in 10, meaning that their product can be written as a compact perturbation of a well-conditioned operator. The self-regularizing property of $\mathcal{T}_{k}$ itself, i.e., the fact that $\mathcal{T}_{k}^{2}$ is a compact perturbation of the identity operator (up to a constant multiplicative factor) on domains with smooth boundaries, was the source of inspiration for efficient preconditioners in BEMs for various integral equations, such as the electric field integral equation (EFIE) [13 15, the combined field integral equations of [16, the regularized combined field integral equations (CFIER) of 17, a single source CFIE for dielectric scattering [18, the Poggio-MillerChan-Harrington-Wu-Tsai (PMCHWT) equation [19], an electric current formulation [20] and the EFIE in a layered medium [21. We emphasize that the cited BEMs are restricted to piecewise homogeneous scatterers, and are not applicable to general heterogeneous domains. The multiplicative nature of the preconditioners in 14, 16, 18, 20 and the specific choice for Buffa-Christiansen (BC) basis functions 22, for the discretization of the $\mathcal{T}_{k}$ operator appearing in the preconditioner, has the benefit that existing method of moments (MoM) algorithms for Rao-Wilton-Glisson (RWG) functions [23] can still be used, albeit on the barycentrically refined mesh.

A wide class of preconditioners for hybrid FEM-BEM formulations exists [24 28], with an important subset of them relying on domain decomposition methods 29, 30. For the first time, a Calderón multiplicative preconditioner is applied to a reduced hybrid FEM-BEM formulation for electromagnetic scattering at a heterogeneous obstacle, and is compatible with existing matrix vector product acceleration schemes, such as the multilevel fast multipole algorithm (MLFMA) 31 34.

This paper is structured as follows: in Section 2 we introduce the electromagnetic Poincaré-Steklov operator $\mathcal{P}$ of a heterogeneous domain and, in line with a similar and well-known result for the electromagnetic PS operator of a homogeneous domain [5, 17, we derive a decomposition in terms of $\mathcal{T}_{k}$, which implies that $\mathcal{T}_{k}$ regularizes $\mathcal{P}$, in a Sobolev space framework, as already remarked in [17]. Inspired by these properties, we construct a Calderón multiplicative preconditioner (CMP) for a Schur complement finite element discretization of $\mathcal{P}$ and investigate its effect on the singular value distribution of the discretized PS operator in Section 3. We present a hybrid FEM-BEM formulation, whose reduced form is amenable to the CMP, in Section 4 , and demonstrate the effectiveness of the preconditioner in the numerical examples of Section 5.

\section{Electromagnetic Poincaré-Steklov Operator of a Heterogeneous Domain}

In this section, the governing time-harmonic Maxwell equations for scattering at a bounded heterogeneous domain are introduced. We define the Poincaré-Steklov operator $\mathcal{P}$ of the heterogeneous domain and derive some of its continuous properties, for the first time, in a Sobolev space framework. An interesting corollary is the regularization of $\mathcal{P}$ by a boundary integral operator $\mathcal{T}_{k}$, sometimes called the electric field integral operator [35, which is frequently used in scattering at perfect electric conducting objects [14, 36, 37. This property hints at a discrete Calderón multiplicative preconditioner for the ill-conditioned discretization of $\mathcal{P}$, which is numerically investigated in Section 3 .

\subsection{Scattering at a Bounded Heterogeneous Domain: Maxwell's equations}

Consider a connected open bounded Lipschitz (see e.g. 38] for a definition) domain $\Omega^{-} \subset \mathbb{R}^{3}$, with boundary $\Gamma$ and external unit normal $\hat{\mathbf{n}}$, representing a heterogeneous isotropic region with permittivity $\epsilon_{1}(\mathbf{r}) \in \mathbb{C}$ and constant permeability $\mu_{0}>0$ (Fig. 1). The domain $\Omega^{-}$is embedded in a homogeneous medium, with permittivity $\epsilon_{0}>0$ and permeability $\mu_{0}$. The structure is excited by an incoming field $\left(\mathbf{e}^{(i)}, \mathbf{h}^{(i)}\right)$ in the homogeneous exterior region $\Omega^{+}=\mathbb{R}^{3} \backslash \overline{\Omega^{-}}$, with $\overline{\Omega^{-}}=\Omega^{-} \cup \Gamma$ being the closure of $\Omega^{-}$. The solution $(\mathbf{e}, \mathbf{h})$ satisfies Maxwell's equations $(1)-\sqrt{2}$ in the frequency domain (angular frequency $\omega$, time 


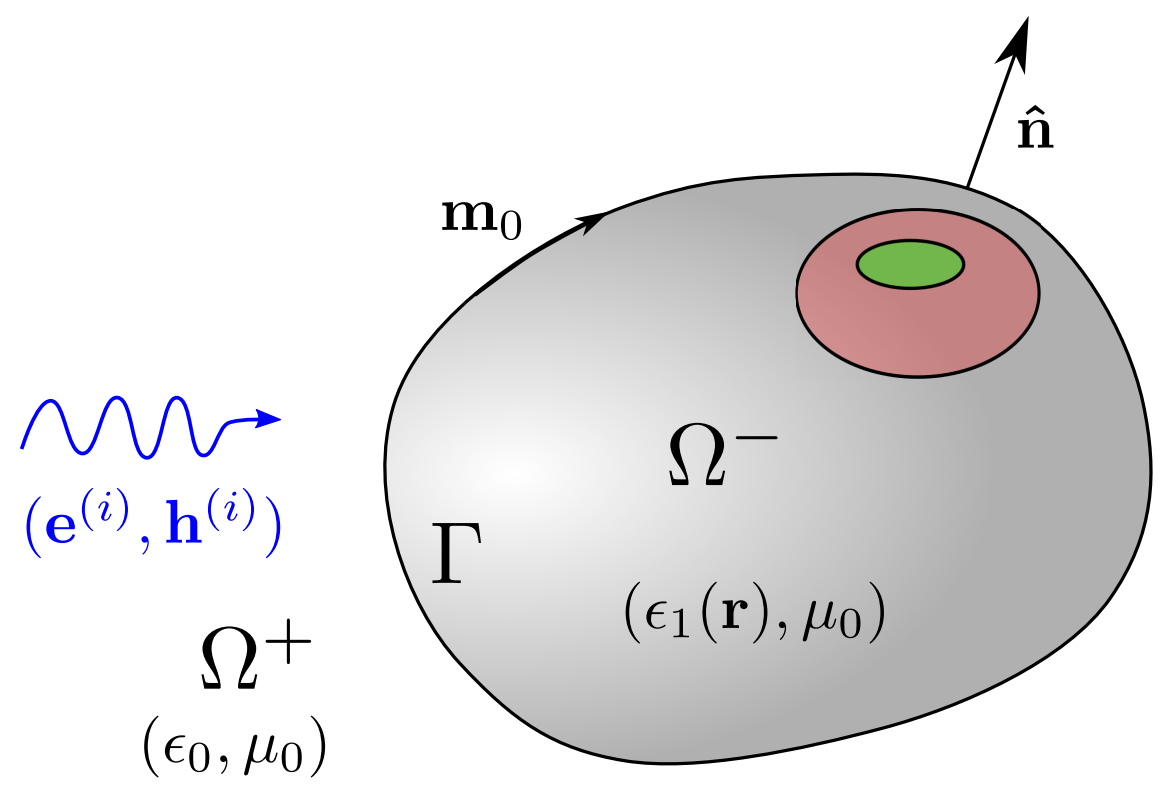

Figure 1: Geometry consisting of a heterogeneous domain $\Omega^{-}$, embedded in a homogeneous space.

factor $\left.e^{j \omega t}\right)$, boundary conditions (3) at $\Gamma$, and a suitable radiation condition (4) [36, 39]:

$$
\begin{aligned}
\nabla \times \mathbf{e}=-j \omega \mu_{0} \mathbf{h}, \nabla \times \mathbf{h}=j \omega \epsilon_{0} \mathbf{e} & \left(\text { in } \Omega^{+}\right), \\
\nabla \times \mathbf{e}=-j \omega \mu_{0} \mathbf{h}, \nabla \times \mathbf{h}=j \omega \epsilon_{1}(\mathbf{r}) \mathbf{e} & \left(\text { in } \Omega^{-}\right), \\
{[\hat{\mathbf{n}} \times \mathbf{e}]_{\Gamma}=\hat{\mathbf{n}} \times \mathbf{e}^{(i)},[\hat{\mathbf{n}} \times \mathbf{h}]_{\Gamma}=\hat{\mathbf{n}} \times \mathbf{h}^{(i)} } & (\text { on } \Gamma), \\
\lim _{|\mathbf{r}| \rightarrow \infty} \mathbf{e} \times \mathbf{r}+\eta|\mathbf{r}| \mathbf{h}=0, &
\end{aligned}
$$

with $\eta=\sqrt{\mu_{0} / \epsilon_{0}}$ the characteristic impedance of the exterior medium and $[\boldsymbol{\psi}]_{\Gamma}=\lim _{\Omega^{-} \ni \mathbf{r} \rightarrow \Gamma} \boldsymbol{\psi}-\lim _{\Omega^{+} \ni \mathbf{r} \rightarrow \Gamma} \boldsymbol{\psi}$, i.e., the jump of $\boldsymbol{\psi}$ across the interface $\Gamma$.

\subsection{Function Spaces and Calderón Projectors}

The precise statement and the proofs of the properties of the PS operator in $₫ 2.3$ make use of the following Sobolev spaces (see [40, 41] for definitions):

$$
\begin{aligned}
\mathbf{L}^{2}\left(\Omega^{-}\right) & =\left(L^{2}\left(\Omega^{-}\right)\right)^{3}, \\
\mathbf{H}^{s}\left(\Omega^{-}\right) & =\left(H^{s}\left(\Omega^{-}\right)\right)^{3}, \\
\mathbf{H}^{s}(\Gamma) & =\left\{\chi \in\left(H^{s}(\Gamma)\right)^{3} \mid \boldsymbol{\chi} \cdot \hat{\mathbf{n}}=0\right\}, \\
\mathbf{H}^{s}\left(\operatorname{curl}, \Omega^{-}\right) & =\left\{\boldsymbol{\phi} \in \mathbf{H}^{s}\left(\Omega^{-}\right) \mid \nabla \times \phi \in \mathbf{H}^{s}\left(\Omega^{-}\right)\right\}, \\
\mathbf{H}^{s}\left(\operatorname{div}_{\Gamma}, \Gamma\right) & =\left\{\boldsymbol{\psi} \in \mathbf{H}^{s}(\Gamma) \mid \operatorname{div}_{\Gamma} \boldsymbol{\psi} \in H^{s}(\Gamma)\right\},
\end{aligned}
$$

with $H^{s}(\Gamma)=W^{s, 2}(\Gamma)$ and $H^{s}\left(\Omega^{-}\right)=W^{s, 2}\left(\Omega^{-}\right)$Hilbert spaces for all $s \in \mathbb{R}$, with the convention that $s=0$ if omitted, i.e., $H=H^{0}=L^{2}$ the space of square integrable densities, and all derivatives considered in distributional sense. Assume that the fields $\mathbf{e}$ and $\mathbf{h}$ belong to $\mathbf{H}\left(\mathrm{curl}, \Omega^{-}\right)$. The trace theorem shows that the tangential traces $\hat{\mathbf{n}} \times \mathbf{e}$ and $\hat{\mathbf{n}} \times \mathbf{h}$ in that case belong to $\mathbf{H}^{-\frac{1}{2}}\left(\operatorname{div}_{\Gamma}, \Gamma\right)$ [1]. Moreover, we define the 
following boundary integral operators $\mathrm{I}^{1}$ in $\mathbf{H}^{-\frac{1}{2}}\left(\operatorname{div}_{\Gamma}, \Gamma\right)$, related to the homogeneous exterior domain $\Omega^{+}$:

$$
\begin{aligned}
& \mathcal{T}_{k}: \mathbf{m} \mapsto \hat{\mathbf{n}} \times j k \int_{\Gamma} G\left(\mathbf{r}-\mathbf{r}^{\prime}\right) \mathbf{m}\left(\mathbf{r}^{\prime}\right) \mathrm{d} S^{\prime}-\hat{\mathbf{n}} \times \frac{1}{j k} \int_{\Gamma} \nabla G\left(\mathbf{r}-\mathbf{r}^{\prime}\right) \nabla^{\prime} \cdot \mathbf{m}\left(\mathbf{r}^{\prime}\right) \mathrm{d} S^{\prime}, \\
& \mathcal{K}_{k}: \mathbf{m} \mapsto \hat{\mathbf{n}} \times \nabla \times \int_{\Gamma} G\left(\mathbf{r}-\mathbf{r}^{\prime}\right) \mathbf{m}\left(\mathbf{r}^{\prime}\right) \mathrm{d} S^{\prime},
\end{aligned}
$$

with $k=\omega \sqrt{\epsilon_{0} \mu_{0}}$ the wavenumber of the homogeneous exterior domain, $\mathbf{r}=(x, y, z)$ and $\mathbf{r}^{\prime}=\left(x^{\prime}, y^{\prime}, z^{\prime}\right)$ position vectors in a Cartesian coordinate system with unit axes $(\hat{\mathbf{x}}, \hat{\mathbf{y}}, \hat{\mathbf{z}}), \nabla=\partial_{x} \hat{\mathbf{x}}+\partial_{y} \hat{\mathbf{y}}+\partial_{z} \hat{\mathbf{z}}, \nabla^{\prime}=$ $\partial_{x^{\prime}} \hat{\mathbf{x}}+\partial_{y^{\prime}} \hat{\mathbf{y}}+\partial_{z^{\prime}} \hat{\mathbf{z}}$ and $G\left(\mathbf{r}-\mathbf{r}^{\prime}\right)=e^{-j k\left|\mathbf{r}-\mathbf{r}^{\prime}\right|} /\left(4 \pi\left|\mathbf{r}-\mathbf{r}^{\prime}\right|\right)$ the outgoing fundamental solution of the scalar Helmholtz equation. Recall that, in a completely homogeneous free space $\mathbb{R}^{3}$, electric and magnetic surface current densities on $\Gamma$, denoted $\mathbf{j}, \mathbf{m} \in \mathbf{H}^{-\frac{1}{2}}\left(\operatorname{div}_{\Gamma}, \Gamma\right)$, respectively, give rise to the following field traces:

$$
\lim _{\Omega^{ \pm} \ni \mathbf{r} \rightarrow \Gamma}\left(\begin{array}{c}
\hat{\mathbf{n}} \times \mathbf{e} \\
\hat{\mathbf{n}} \times \mathbf{h}
\end{array}\right)=\mathbb{P}^{ \pm}\left(\begin{array}{c}
\mathbf{m} \\
\mathbf{j}
\end{array}\right), \mathbb{P}^{ \pm}=\left(\begin{array}{cc}
\mathcal{K}_{k} \pm \frac{1}{2} & -\eta \mathcal{T}_{k} \\
\mathcal{T}_{k} / \eta & \mathcal{K}_{k} \pm \frac{1}{2}
\end{array}\right)
$$

with $\mathbb{P}^{+}$and $\mathbb{P}^{-}$the exterior and interior Calderón projectors, respectively 37, 41. The projector property $\left(\mathbb{P}^{ \pm}\right)^{2}=\mathbb{P}^{ \pm}$immediately implies the so-called Calderón identities

$$
\begin{aligned}
\mathcal{T}_{k} \mathcal{K}_{k}+\mathcal{K}_{k} \mathcal{T}_{k} & =0 \\
\mathcal{K}_{k}^{2}-\mathcal{T}_{k}^{2} & =\frac{1}{4} .
\end{aligned}
$$

Lemma 2.1. $\mathcal{K}_{k}$ is compact in $\mathbf{H}^{-\frac{1}{2}}\left(\operatorname{div}_{\Gamma}, \Gamma\right)$ on smooth $\Gamma$.

Proof. This follows from the fact that $\mathcal{K}_{k}: \mathbf{H}^{-\frac{1}{2}}\left(\operatorname{div}_{\Gamma}, \Gamma\right) \rightarrow \mathbf{H}^{\frac{1}{2}}\left(\operatorname{div}_{\Gamma}, \Gamma\right)$ is continuous on smooth $\Gamma$ (as proven in [41, Lemma 11]) and the compact embedding $\mathbf{H}^{\frac{1}{2}}\left(\operatorname{div}_{\Gamma}, \Gamma\right) \subset \subset \mathbf{H}^{-\frac{1}{2}}\left(\operatorname{div}_{\Gamma}, \Gamma\right)$.

\subsection{Definition and Properties of the Poincaré-Steklov Operator}

The electromagnetic Poincaré-Steklov operator, denoted $\mathcal{P}$, is defined in this contribution ${ }^{2}$ as the boundary operator that maps the tangential electric field trace $\hat{\mathbf{n}} \times\left.\mathbf{e}\right|_{\Gamma}$ of a solution to the sourceless Maxwell equations inside $\Omega^{-}$onto the corresponding tangential magnetic field trace $\hat{\mathbf{n}} \times\left.\mathbf{h}\right|_{\Gamma}$.

Definition 2.1 (Poincaré-Steklov Operator). $\mathcal{P}: \mathbf{H}^{-\frac{1}{2}}\left(\operatorname{div}_{\Gamma}, \Gamma\right) \rightarrow \mathbf{H}^{-\frac{1}{2}}\left(\operatorname{div}_{\Gamma}, \Gamma\right): \hat{\mathbf{n}} \times\left.\mathbf{e}\right|_{\Gamma} \mapsto \hat{\mathbf{n}} \times\left.\mathbf{h}\right|_{\Gamma}$, with

$$
\nabla \times \mathbf{e}=-j \omega \mu_{0} \mathbf{h}, \nabla \times \mathbf{h}=j \omega \epsilon_{1} \mathbf{e} \quad\left(\text { in } \Omega^{-}\right) .
$$

In the sequel, we prove a specific decomposition of $\mathcal{P}$ (under certain restrictions on $\Gamma$ and $\epsilon_{1}$ ). This decomposition explains the dense mesh discretization breakdown of a finite-dimensional discretization of $\mathcal{P}$, denoted $\mathrm{P} \in \mathbb{C}^{n \times n}$, implying that the condition number of $\mathrm{P}$ grows without bound for an increasing number $n$ of basis functions, as observed in Section 3 .

Theorem 2.2 (Decomposition of $\mathcal{P}$ ). Suppose the boundary $\Gamma$ is smooth, and the permittivity $\epsilon_{1} \in C^{1}\left(\mathbb{R}^{3}\right)$, with $\epsilon_{1}(\mathbf{r}) \neq 0 \forall \mathbf{r} \in \mathbb{R}^{3}$ and $\left.\epsilon_{1}\right|_{\Omega^{+}}=\epsilon_{0}$. Let $k>0$ not be a resonant wavenumber of $\Omega^{-}$. Then

$$
\mathcal{P}=-\frac{2}{\eta} \mathcal{T}_{k}(1+\mathcal{C})
$$

with $\mathcal{C}$ compact in $\mathbf{H}^{-\frac{1}{2}}\left(\operatorname{div}_{\Gamma}, \Gamma\right)$.

\footnotetext{
${ }^{1}$ all integrals are considered in Cauchy principal value sense, i.e., as the limit for $\delta \rightarrow 0$ of the integrals over the surface $\Gamma \backslash \Gamma_{\delta}$, with $\Gamma_{\delta}$ the intersection of $\Gamma$ with a ball with radius $\delta$, centered around the singularity $\mathbf{r} \in \Gamma$ of the integrands [36] ${ }^{2}$ note that $\hat{\mathbf{n}} \times \mathcal{P}$ is called the Poincaré-Steklov operator in 4
} 
Proof. Consider a magnetic current density $\mathbf{m} \in \mathbf{H}^{-\frac{1}{2}}\left(\operatorname{div}_{\Gamma}, \Gamma\right)$ that flows on $\Gamma$ and generates electric and magnetic fields $\mathbf{e}$ and $\mathbf{h}$ inside $\Omega^{-}$. With $\epsilon_{1}=\epsilon_{0}(1+\widetilde{\epsilon})$, Maxwell's equations 15 can be written as

$$
\begin{aligned}
& \nabla \times \mathbf{e}=-j \omega \mu_{0} \mathbf{h}, \\
& \nabla \times \mathbf{h}=j \omega \epsilon_{0} \mathbf{e}+\mathbf{j}_{c},
\end{aligned}
$$

where $\mathbf{j}_{c}=j \omega \epsilon_{0} \tilde{\epsilon} \mathbf{e}$ is the so-called contrast current. Consider the following operators, with $G\left(\mathbf{r}-\mathbf{r}^{\prime}\right)=$ $e^{-j k\left|\mathbf{r}-\mathbf{r}^{\prime}\right|} /\left(4 \pi\left|\mathbf{r}-\mathbf{r}^{\prime}\right|\right):$

$$
\begin{aligned}
\mathcal{A} & : \mathbf{H}^{-\frac{1}{2}}\left(\operatorname{div}_{\Gamma}, \Gamma\right) \rightarrow \mathbf{H}\left(\operatorname{curl}, \Omega^{-}\right): \mathbf{m} \mapsto \nabla \times \int_{\Gamma} G\left(\mathbf{r}-\mathbf{r}^{\prime}\right) \mathbf{m}\left(\mathbf{r}^{\prime}\right) \mathrm{d} S^{\prime}, \\
\mathcal{B} & : \mathbf{H}\left(\operatorname{curl}, \Omega^{-}\right) \rightarrow \mathbf{H}\left(\operatorname{curl}, \Omega^{-}\right): \mathbf{e} \mapsto k^{2} \int_{\Omega^{-}} G\left(\mathbf{r}-\mathbf{r}^{\prime}\right) \widetilde{\epsilon}\left(\mathbf{r}^{\prime}\right) \mathbf{e}\left(\mathbf{r}^{\prime}\right) \mathrm{d} V^{\prime} \\
& +\nabla \int_{\Omega^{-}} G\left(\mathbf{r}-\mathbf{r}^{\prime}\right) \frac{1}{1+\widetilde{\epsilon}} \nabla^{\prime} \widetilde{\epsilon} \cdot \mathbf{e} \mathrm{d} V^{\prime} .
\end{aligned}
$$

The operator $\mathcal{A}$ maps the magnetic current density onto the electric field it generates in a homogeneous region $\Omega^{-}$(with constant permittivity $\epsilon_{0}$ ). It follows from [41, Theorem 5] that $\mathcal{A}$ is bounded. Taking the divergence of equation 17 shows that $\nabla \cdot[\widetilde{\epsilon} \mathbf{e}]=\frac{1}{1+\widetilde{\epsilon}} \nabla \widetilde{\epsilon} \cdot \mathbf{e}$, so that the operator $\mathcal{B}$ maps this incoming electric field onto the electric field generated by the contrast current $j \omega \epsilon_{0} \tilde{\epsilon}$ e. The assumption $\epsilon_{1} \in C^{1}\left(\mathbb{R}^{3}\right)$ implies that $\left|\epsilon_{1}\right|$ and $\left|\nabla \epsilon_{1}\right|$ are continuous on the compact set $\overline{\Omega^{-}}$and, hence, by the extreme value theorem, they attain a minimum and maximum in $\overline{\Omega^{-}}$, implying that $\left|\nabla \epsilon_{1}\right|=|\nabla \widetilde{\epsilon}|$ and $|\tilde{\epsilon}|$ are bounded in $\Omega^{-}$. Moreover, by the assumption that $\epsilon_{1}(\mathbf{r}) \neq 0 \forall \mathbf{r} \in \mathbb{R}^{3}$, the attained minimal value of $\left|\epsilon_{1}\right|=\left|\epsilon_{0}\right| \cdot|1+\tilde{\epsilon}|$ is bigger than zero, implying that $\exists c>0:|1+\widetilde{\epsilon}| \geq c$ in $\Omega^{-}$. Therefore, $|\nabla \widetilde{\epsilon} /(1+\widetilde{\epsilon})|$ is also bounded in $\Omega^{-}$, implying that $\mathcal{B}$ is a weakly singular integral operator, and thus compact in $\mathbf{L}^{2}\left(\Omega^{-}\right)$. Moreover, $\nabla \times \mathcal{B}$ is also a weakly singular integral operator, and thus compact in $\mathbf{L}^{2}\left(\Omega^{-}\right)\left[42\right.$. This implies that $\mathcal{B}$ is compact in $\mathbf{H}\left(\right.$ curl, $\left.\Omega^{-}\right)$ and that, as the heterogeneous scattering problem has a unique solution [43, Section 9.2], $(1-\mathcal{B})^{-1}$ is bounded in $\mathbf{H}\left(\right.$ curl, $\left.\Omega^{-}\right)$. In the last step, we transform the scattered electric field back to the boundary with the trace operator $\gamma_{\mathbf{t}}: \mathbf{H}\left(\mathrm{curl}, \Omega^{-}\right) \rightarrow \mathbf{H}^{-\frac{1}{2}}\left(\operatorname{div}_{\Gamma}, \Gamma\right): \mathbf{u} \mapsto \hat{\mathbf{n}} \times\left.\mathbf{u}\right|_{\Gamma}$. The continuity of this operator is well-established [4]. This leads to the following expression for the scattered electric field, generated by the contrast currents, for the given incoming field $\mathcal{A m}$ :

$$
\hat{\mathbf{n}} \times \mathbf{e}_{c}=\gamma_{\mathbf{t}} \mathcal{B}(1-\mathcal{B})^{-1} \mathcal{A} \mathbf{m},
$$

with $\mathcal{Q}=\gamma_{\mathbf{t}} \mathcal{B}(1-\mathcal{B})^{-1} \mathcal{A}$ compact in $\mathbf{H}^{-\frac{1}{2}}\left(\operatorname{div}_{\Gamma}, \Gamma\right)$, as $\mathcal{B}$ is compact and $\mathcal{A},(1-\mathcal{B})^{-1}$ and $\gamma_{\mathbf{t}}$ are all bounded. The scattered magnetic field follows from the observation that the scattered field is a solution of Maxwell's equations (1) in $\Omega^{+}$satisfying the radiation condition (3). This means that its traces belong to the kernel of the interior Calderón projector $\mathbb{P}^{-}[41$, Theorem 8], defined in $[12$, leading to

$$
\hat{\mathbf{n}} \times \mathbf{h}_{c}=\frac{1}{\eta} \mathcal{T}_{k}\left(\mathcal{K}_{k}+\frac{1}{2}\right)^{-1} \mathcal{Q} \mathbf{m} .
$$

To conclude, the total tangential electric and magnetic fields on the boundary (by taking the limit from the interior region $\Omega^{-}$) can be written as a sum of the incoming fields, generated by $\mathbf{m}$, and scattered fields, generated by the contrast currents, as:

$$
\begin{aligned}
\hat{\mathbf{n}} \times \mathbf{e} & =\left[\mathcal{K}_{k}-\frac{1}{2}+\mathcal{Q}\right] \mathbf{m}, \\
\hat{\mathbf{n}} \times \mathbf{h} & =\left[\frac{1}{\eta} \mathcal{T}_{k}+\frac{1}{\eta} \mathcal{T}_{k}\left(\mathcal{K}_{k}+\frac{1}{2}\right)^{-1} \mathcal{Q}\right] \mathbf{m} .
\end{aligned}
$$

This yields the following expression for the Poincaré-Steklov operator:

$$
\mathcal{P}=\frac{1}{\eta} \mathcal{T}_{k}\left[1+\left(\mathcal{K}_{k}+\frac{1}{2}\right)^{-1} \mathcal{Q}\right]\left[\mathcal{K}_{k}-\frac{1}{2}+\mathcal{Q}\right]^{-1} .
$$


In case of a homogeneous interior domain, $\widetilde{\epsilon}=0$ implies that $\mathcal{Q}=0$ and (24) reduces to the well-known result $\mathcal{P}=\frac{1}{\eta} \mathcal{T}_{k}\left[\mathcal{K}_{k}-\frac{1}{2}\right]^{-1}[17]$.

For smooth $\Gamma$, the operator $\mathcal{K}_{k}$ is compact (see Lemma 2.1). Note that the inverses $\left(\mathcal{K}_{k}+\frac{1}{2}\right)^{-1}$ and $\left(\mathcal{K}_{k}-\frac{1}{2}+\mathcal{Q}\right)^{-1}$ in 24 exist, because we assume that $k>0$ is not a resonant wavenumber of the heterogeneous (permittivity $\epsilon_{1}$ ) or homogeneous (constant permittivity $\epsilon_{0}$ ) domain $\Omega^{-}$. By the compactness of $\mathcal{K}_{k}$ and $\mathcal{Q}$, these inverses are also bounded. Combining the compactness of $\mathcal{Q}$ and the boundedness of $\left(\mathcal{K}_{k}+\frac{1}{2}\right)^{-1}$ and $\left(\mathcal{K}_{k}-\frac{1}{2}+\mathcal{Q}\right)^{-1}$ leads to the proposition.

This implies that $\mathcal{T}_{k}$ regularizes $\mathcal{P}$, as indicated by the following corollary, in correspondence with the same property for the PS operator of a homogeneous domain [5, 17.

Corollary 2.1. Under the stated assumptions, $\mathcal{T}_{k} \mathcal{P}=\frac{1}{2 \eta}+\mathcal{D}$, with $\mathcal{D}$ compact in $\mathbf{H}^{-\frac{1}{2}}\left(\operatorname{div}_{\Gamma}, \Gamma\right)$.

Proof. This follows directly from the decomposition of $\mathcal{P}$ in Theorem 2.2 together with the Calderón identity (14) and Lemma 2.1

Moreover, every operator $\mathcal{T}_{k^{\prime}}$ (with wavenumber $k^{\prime}>0$ ) can be used to regularize $\mathcal{P}$, as indicated by the following corollary. It explains the successful application of the Calderón multiplicative preconditioner 43 . to the hybrid system (42) for scattering at a heterogeneous domain with $\left.\epsilon_{1}\right|_{\Gamma} \neq \epsilon_{0}$ (i.e., with a discontinuous jump of the permittivity at the boundary), as demonstrated in the examples of Section 5 .

Corollary 2.2. Under the stated assumptions, $\mathcal{T}_{k^{\prime}} \mathcal{P}=\frac{1}{2 \eta}\left(\frac{k^{\prime}}{k} \Pi_{\nabla_{\Gamma}}+\frac{k}{k^{\prime}} \Pi_{\mathrm{curl}_{\Gamma}}\right)+\mathcal{D}^{\prime}$, with $\mathcal{D}^{\prime}$ compact in $\mathbf{H}^{-\frac{1}{2}}\left(\operatorname{div}_{\Gamma}, \Gamma\right) \forall k^{\prime}>0, \Pi_{\nabla_{\Gamma}}$ and $\Pi_{\mathrm{curl}_{\Gamma}}$ orthogonal projectors and $\Pi_{\nabla_{\Gamma}}+\Pi_{\mathrm{curl}_{\Gamma}}=1$ in $\mathbf{H}^{-\frac{1}{2}}\left(\operatorname{div}_{\Gamma}, \Gamma\right)$.

Proof. The proposition follows directly from Theorem 2.2 and the fact that $\mathcal{T}_{k^{\prime}} \mathcal{T}_{k}$ is a compact perturbation of $-\frac{1}{4}\left(\frac{k^{\prime}}{k} \Pi_{\nabla_{\Gamma}}+\frac{k}{k^{\prime}} \Pi_{\text {curl }_{\Gamma}}\right)$ for $k^{\prime}>0\left[17\right.$, with $\Pi_{\nabla_{\Gamma}}$ and $\Pi_{\mathrm{curl}_{\Gamma}}$ the orthogonal projectors associated to the Helmholtz decomposition of $\mathbf{H}^{-\frac{1}{2}}\left(\operatorname{div}_{\Gamma}, \Gamma\right)$ and defined in [17].

\section{Discretized Poincaré-Steklov Operator}

The numerical simulation of the time-harmonic Maxwell equations (2) in a heterogeneous domain may typically involve the finite element method (FEM) [4, 45]. The hereafter introduced discretization of the PS operator, denoted $\mathrm{P}$, originates from the Schur complement of the FEM matrix w.r.t. the boundary degrees of freedom. Its conditioning, including dense mesh discretization breakdown and the effect of the proposed Calderón multiplicative preconditioner on its singular values and condition number, stem from the derived theorems in $\$ 2.3$ and are investigated as a function of the mesh density.

\subsection{Finite Element Schur Complement Discretization}

Consider the electric field wave equation in the heterogeneous domain (25), which follows directly from (2), and its weak formulation (26).

$$
\begin{array}{r}
\nabla \times \nabla \times \mathbf{e}-\omega^{2} \mu_{0} \epsilon_{1}(\mathbf{r}) \mathbf{e}=0 \\
\int_{\Omega^{-}} \boldsymbol{\psi} \cdot\left(\nabla \times \nabla \times \mathbf{e}-\omega^{2} \mu_{0} \epsilon_{1}(\mathbf{r}) \mathbf{e}\right) \mathrm{d} V=0
\end{array}
$$

By a suitable Green's theorem [36, 26] reduces to

$$
\int_{\Omega^{-}}\left((\nabla \times \boldsymbol{\psi}) \cdot(\nabla \times \mathbf{e})-\omega^{2} \mu_{0} \epsilon_{1}(\mathbf{r}) \boldsymbol{\psi} \cdot \mathbf{e}\right) \mathrm{d} V=\int_{\Gamma}(\hat{\mathbf{n}} \times \boldsymbol{\psi}) \cdot(\nabla \times \mathbf{e}) \mathrm{d} S .
$$

Electric fields e $\in \mathbf{H}\left(\mathrm{curl}, \Omega^{-}\right)$that satisfy $\sqrt{27)} \forall \boldsymbol{\psi} \in \mathbf{H}\left(\mathrm{curl}, \Omega^{-}\right)$are exact solutions to the Maxwell equations in the heterogeneous domain. In the finite element method, e and $\boldsymbol{\psi}$ are restricted to a finitedimensional finite element space $\mathcal{V}^{h} \subset \mathbf{H}\left(\mathrm{curl}, \Omega^{-}\right)$, with $h>0$ denoting the characteristic size of the mesh 
elements, i.e., we are interested in approximate solutions e $\in \mathcal{V}^{h}$ such that (27) holds $\forall \boldsymbol{\psi} \in \mathcal{V}^{h}$. Let us denote the subspace of finite elements with vanishing tangential trace as $\mathcal{V}_{i}^{h}=\left\{\mathbf{e} \in \mathcal{V}^{h}: \hat{\mathbf{n}} \times\left.\mathbf{e}\right|_{\Gamma}=0\right\}$, which contains the finite elements associated to the $N_{i}=\operatorname{dim}\left(\mathcal{V}_{i}^{h}\right)$ interior degrees of freedom, and a subspace denoted $\mathcal{V}_{b}^{h}$, containing the finite elements associated to the $N_{b}=\operatorname{dim}\left(\mathcal{V}_{b}^{h}\right)$ boundary degrees of freedom and isomorphic to the quotient space $\mathcal{V}^{h} / \mathcal{V}_{i}^{h}$. This implies the following splitting, with $\oplus$ the direct sum:

$$
\mathcal{V}^{h}=\mathcal{V}_{i}^{h} \oplus \mathcal{V}_{b}^{h}
$$

Denote the components of $\mathbf{e} \in \mathcal{V}^{h}$ as $\mathbf{e}_{i} \in \mathcal{V}_{i}^{h}$ and $\mathbf{e}_{b} \in \mathcal{V}_{b}^{h}$, with $\mathbf{e}=\mathbf{e}_{i}+\mathbf{e}_{b}$ (and, mutatis mutandis, for $\boldsymbol{\psi})$. With the bases $\left\{\mathbf{b}_{i}^{j}\right\}_{1 \leq j \leq N_{i}}$ and $\left\{\mathbf{b}_{b}^{j}\right\}_{1 \leq j \leq N_{b}}$ of characteristic functions corresponding to the degrees of freedom in $\mathcal{V}_{i}^{h}$ and $\mathcal{V}_{b}^{h}$, respectively, denote $\bar{e}_{i} \in \mathbb{C}^{N_{i}}$ such that $\mathbf{e}_{i}=\sum_{j}\left(\bar{e}_{i}\right)_{j} \mathbf{b}_{i}^{j}$ (and likewise for $\mathbf{e}_{b}$ ). This reduces (27), with $\mathbf{e}, \boldsymbol{\psi} \in \mathcal{V}^{h}$, to the following linear system:

$$
\left(\begin{array}{cc}
\mathrm{A}_{i i} & \mathrm{~A}_{i b} \\
\mathrm{~A}_{b i} & \mathrm{~A}_{b b}
\end{array}\right)\left(\begin{array}{c}
\bar{e}_{i} \\
\bar{e}_{b}
\end{array}\right)=\left(\begin{array}{c}
\overline{0} \\
\bar{a}_{b}
\end{array}\right)
$$

The elements of the sparse complex symmetric system matrix and the right hand side vector are given by

$$
\begin{aligned}
\left(\mathrm{A}_{m n}\right)_{k l} & =\int_{\Omega^{-}}\left[\left(\nabla \times \mathbf{b}_{m}^{k}\right) \cdot\left(\nabla \times \mathbf{b}_{n}^{l}\right)-\omega^{2} \mu_{0} \epsilon_{1}(\mathbf{r}) \mathbf{b}_{m}^{k} \cdot \mathbf{b}_{n}^{l}\right] \mathrm{d} V, \quad m, n \in\{i, b\}, \\
\left(\bar{a}_{b}\right)_{k} & =\int_{\Gamma}\left(\hat{\mathbf{n}} \times \mathbf{b}_{b}^{k}\right) \cdot(\nabla \times \mathbf{e}) \mathrm{d} S .
\end{aligned}
$$

Definition 3.1 (Finite Element Discretization of $\mathcal{P}$ ). If $\mathrm{A}_{i i}$ is invertible (i.e., away from Dirichlet and Neumann frequencies of the heterogeneous domain), the finite element discretization of $\mathcal{P}$ is defined as

$$
\mathrm{P}=\frac{1}{j \omega \mu_{0}}\left(\mathrm{~A}_{b b}-\mathrm{A}_{b i} \mathrm{~A}_{i i}^{-1} \mathrm{~A}_{i b}\right)
$$

This immediately implies that

$$
\mathrm{P} \bar{e}_{b}=\frac{1}{j \omega \mu_{0}} \bar{a}_{b} .
$$

Lemma 3.1 provides a connection between $\mathrm{P}$ and $\mathcal{P}$.

Lemma 3.1. If $\mathbf{e} \in \mathcal{V}^{h}$ is an exact solution to the wave equation 25), then $\left(\mathrm{P} \bar{e}_{b}\right)_{k}=\int_{\Gamma} \mathbf{b}_{b}^{k} \cdot \mathcal{P}\left(\hat{\mathbf{n}} \times \mathbf{e}_{b}\right) \mathrm{d} S$.

Proof. If $\mathbf{e} \in \mathcal{V}^{h}$ satisfies 25 , then $\mathbf{e}$ and $\mathbf{h}=\frac{j}{\omega \mu_{0}} \nabla \times \mathbf{e}$ satisfy $\sqrt{15}$, implying that $\hat{\mathbf{n}} \times\left(\frac{j}{\omega \mu_{0}} \nabla \times \mathbf{e}\right)=$ $\mathcal{P}(\hat{\mathbf{n}} \times \mathbf{e})=\mathcal{P}\left(\hat{\mathbf{n}} \times \mathbf{e}_{b}\right)$. By 33 and 31 we get

$$
\left(\mathrm{P} \bar{e}_{b}\right)_{k}=\frac{1}{j \omega \mu_{0}}\left(\bar{a}_{b}\right)_{k}=\int_{\Gamma} \mathbf{b}_{b}^{k} \cdot\left(\hat{\mathbf{n}} \times\left(\frac{j}{\omega \mu_{0}} \nabla \times \mathbf{e}\right)\right) \mathrm{d} S .
$$

For an exact solution e to the wave equation 25 that lies not necessarily in $\mathcal{V}^{h}$, it can be shown that $\mathrm{P}$ converges to the continuous operator $\mathcal{P}$, in the sense that

$$
\left(\mathrm{P} \bar{e}_{b}\right)_{m} \underset{h \rightarrow 0}{\longrightarrow} \int_{\Gamma} \mathbf{b}_{b}^{m} \cdot \mathcal{P}(\hat{\mathbf{n}} \times \mathbf{e}) \mathrm{d} S,
$$

if $\Pi^{h}(\hat{\mathbf{n}} \times \mathbf{e})=\sum_{k}\left(\bar{e}_{b}\right)_{k}\left(\hat{\mathbf{n}} \times \mathbf{b}_{b}^{k}\right)$ is an interpolation of $\hat{\mathbf{n}} \times \mathbf{e}$ onto the set of functions $\hat{\mathbf{n}} \times \mathbf{b}_{b}^{k}$ (which depend on $h$ ) with $\Pi^{h}(\hat{\mathbf{n}} \times \mathbf{e}) \underset{h \rightarrow 0}{\longrightarrow} \hat{\mathbf{n}} \times \mathbf{e}$, and $\underset{h \rightarrow 0}{\longrightarrow}$ denoting convergence if the mesh size goes to zero (for a family of regular meshes [41, 44]). Therefore, $\mathrm{P}$ can be called an approximation of a Galerkin discretization of $\mathcal{P}$ w.r.t. the boundary functions $\mathbf{b}_{b}^{k}$. 


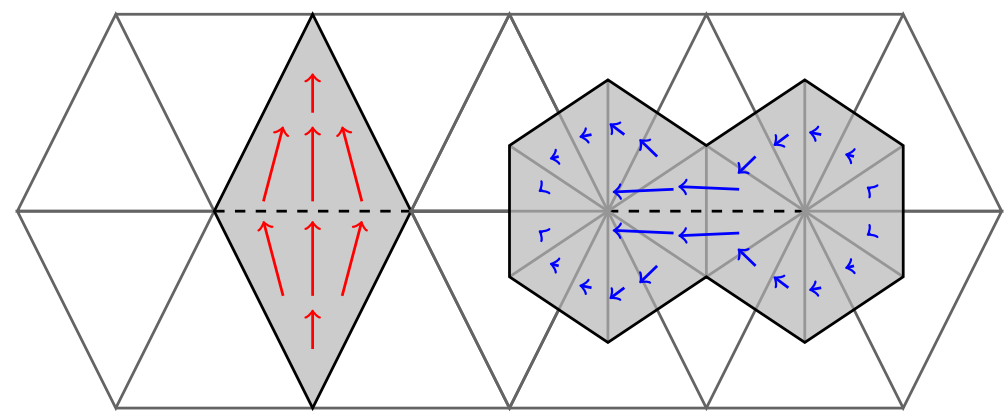

Figure 2: Divergence conforming Rao-Wilton-Glisson (left, $\hat{\mathbf{n}} \times \mathbf{b}_{m}^{k}$ ) and Buffa-Christiansen (right, $\mathbf{d}^{n}$ ) basis functions associated to the edges (in dotted line) of the triangulated surface mesh $\Gamma_{h}$.

\subsection{Basis Functions}

In this contribution, we approximate the domain $\Omega^{-}$as a partition of tetrahedra, with $h>0$ the maximum edge length. Consequently, the boundary $\Gamma_{h}$ is a triangulated surface. Moreover, we choose $\mathcal{V}^{h}$ as the space of lowest order Nédélec curl conforming elements [46, with basis functions $\mathbf{b}_{i}^{m}$ and $\mathbf{b}_{b}^{n}$ associated to the tangential electric field degrees of freedom on the internal and boundary edges, respectively. This implies in particular that the divergence conforming basis function $\hat{\mathbf{n}} \times \mathbf{b}_{b}^{m}$, i.e., the rotated trace of the Nédélec boundary edge element $\mathbf{b}_{b}^{m}$, corresponds to a lowest order Rao-Wilton-Glisson (RWG) function 23. (see Fig. 2). For the discretization of the boundary operators $\mathcal{T}_{k}$, which arises in the Calderón preconditioner, and $\mathcal{K}_{k}$, which arises in the hybrid formulation of Section 4, we use the same triangular surface mesh $\Gamma_{h}$ and define two types of basis functions on this mesh, associated with the edges (see Fig. 2). First, we use the RWG basis functions $\hat{\mathbf{n}} \times \mathbf{b}_{b}^{k} \in \mathbf{H}^{-\frac{1}{2}}\left(\operatorname{div}_{\Gamma}, \Gamma\right)$ for a Galerkin discretization of $\mathcal{T}_{k}$, denoted $\mathrm{T}$.

$$
(\mathbf{T})_{m n}=\int_{\Gamma} \mathbf{b}_{b}^{m} \cdot \mathcal{T}_{k}\left(\hat{\mathbf{n}} \times \mathbf{b}_{b}^{n}\right) \mathrm{d} S
$$

The discretization of the left $\mathcal{T}_{k}$ operator in the well-conditioned operator products $\mathcal{T}_{k} \mathcal{T}_{k}$ and $\mathcal{T}_{k} \mathcal{P}$, which arise in the hybrid formulation of Section 4, makes use of the basis of Buffa-Christiansen (BC) functions 22. $\mathbf{d}^{n}$, defined on the barycentrically refined mesh and dual to the RWG basis functions (Fig. 2). Denote the Galerkin discretization of $\mathcal{T}_{k}$ w.r.t. the $\mathrm{BC}$ basis $\mathbf{d}^{n} \in \mathbf{H}^{-\frac{1}{2}}\left(\operatorname{div}_{\Gamma}, \Gamma\right)$ as $\mathrm{T}_{\mathrm{bc}}$.

$$
\left(\mathrm{T}_{\mathrm{bc}}\right)_{m n}=\int_{\Gamma}\left(\hat{\mathbf{n}} \times \mathbf{d}^{m}\right) \cdot \mathcal{T}_{k} \mathbf{d}^{n} \mathrm{~d} S
$$

The mixed discretization of the operator $\mathcal{K}_{k}$, with RWG basis functions and $\hat{\mathbf{n}} \times \mathrm{BC}$ test functions, is denoted K.

$$
(\mathrm{K})_{m n}=\int_{\Gamma}\left(\hat{\mathbf{n}} \times \mathbf{d}^{m}\right) \cdot \mathcal{K}_{k}\left(\hat{\mathbf{n}} \times \mathbf{b}_{b}^{n}\right) \mathrm{d} S
$$

The mixed Gram matrix G links the RWG and BC bases.

$$
(\mathrm{G})_{m n}=\int_{\Gamma} \mathbf{b}_{b}^{m} \cdot \mathbf{d}^{n} \mathrm{~d} S
$$

\subsection{Dense Mesh Discretization Breakdown}

Fig. 3 shows that the condition number of $P$ (for the example of the Luneburg lens of $\$ 5.2$ is already considerable for the moderate mesh size $h=0.05 \lambda$, and continues to grow monotonically if the mesh size decreases. This undesirable ill-conditioning, which is called dense mesh discretization breakdown, severely hampers both the accuracy (due to an increased sensitivity of the solution vector on the matrix and the 


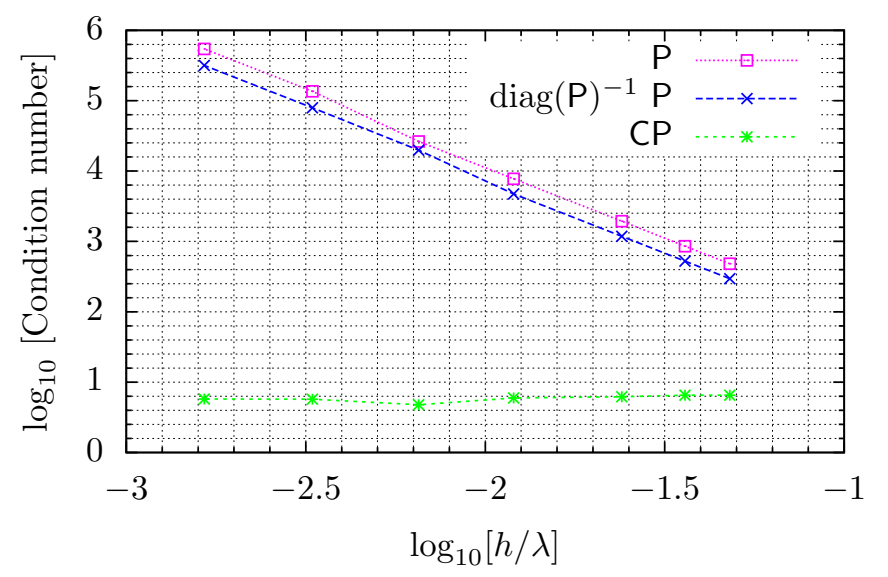

Figure 3: Condition number of $\mathrm{P}$, with diagonal preconditioner $\operatorname{diag}(\mathrm{P})^{-1}$ and Calderón preconditioner $\mathrm{C}$, versus the relative mesh element size $h / \lambda$ for a Luneburg lens.

right hand side accuracies) and convergence speed of iterative solvers [47. The latter will be illustrated in the numerical examples of Section 5 .

The breakdown can be explained by the decomposition of $\mathcal{P}$ in Theorem 2.2 . Note that $\mathcal{P}$ is proportional, up to a compact term $\left(\operatorname{as} \mathcal{T}_{k}\right.$ is bounded in $\left.\mathbf{H}^{-\frac{1}{2}}\left(\operatorname{div}_{\Gamma}, \Gamma\right)\right)$, to the Electric Field Integral Operator $\left(\right.$ EFIO) $\mathcal{T}_{k}$, whose Galerkin discretization is known to suffer from dense mesh discretization breakdown [14. The compact term does not influence the singular value distribution of $\mathcal{P}$ significantly, as its singular values accumulate at 0 . Indeed, the singular value distributions of $\mathrm{T}$ and $\mathrm{P}$ for a dense mesh $\left(h / \lambda=1.7 \cdot 10^{-3}, N_{b}=4521\right.$ unknowns) are very similar (Fig. 4p, with the well-known two branches of singular values accumulating at 0 and $\infty$ (for $N_{b} \rightarrow \infty$ ) clearly visible [14. As a consequence, the $h^{-2}$ behavior of the condition number of T 48] is also encountered in the discretization $\mathrm{P}$ (see Fig. 3).

\subsection{Calderón Multiplicative Preconditioner}

Our goal is to propose a multiplicative preconditioner $C \in \mathbb{C}^{N_{b} \times N_{b}}$ such that the condition number of CP is uniformly bounded as a function of $N_{b}$, i.e., such that $C$ cures $\mathrm{P}$ of dense mesh discretization breakdown. Corollary 2.1 states that the operator product $2 \eta \mathcal{T}_{k} \mathcal{P}$ is the sum of a compact operator (whose singular values accumulate at zero) and the identity operator, implying that $\mathcal{T}_{k} \mathcal{P}$ is well-conditioned in $\mathbf{H}^{-\frac{1}{2}}\left(\operatorname{div}_{\Gamma}, \Gamma\right)$ (its singular values accumulate at $\frac{1}{2 \eta}$ ).

Inspired by these observations, we are looking for $C$ such that $C P$ is a discretization of the continuous operator $\mathcal{T}_{k} \mathcal{P}$. The specific expression for $\mathrm{C}$ described hereafter corresponds to the multiplicative preconditioner of the EFIO $\mathcal{T}_{k}$ based on RWG and BC basis functions for the discretization of the two operators in the product $\mathcal{T}_{k} \mathcal{T}_{k}[14,22$. This has the additional advantage that $\mathrm{C}$ is also a preconditioner for $\mathrm{T}$, which will be exploited in the construction of an efficient preconditioner for the proposed hybrid formulation in Section 4. In line with [14] and with contributions on similar preconditioning strategies for other operators [16 21, 49, 50, we call C a Calderón multiplicative preconditioner. Similar to [14], we choose

$$
\mathrm{C}=\mathrm{T}_{\mathrm{bc}} \mathrm{G}^{-1} \text {. }
$$

By (35), $\mathrm{P}$ is (an approximation of) a Galerkin discretization of $\mathcal{P}$ with RWG basis functions $\hat{\mathbf{n}} \times \mathbf{b}_{b}^{m}$. By (36), $\mathrm{T}_{\mathrm{bc}}$ is a Galerkin discretization of $\mathcal{T}_{k}$ with BC basis functions $\mathbf{d}^{m}$. It has been proven that the condition number of the sparse mixed Gram G, linking RWG and BC functions, is uniformly bounded as a function of $N_{b}$ (for regular meshes) [22, and is small in practice [14, which ensures a fast iterative solution of its inverse. The choice of $\mathrm{BC}$ functions for the discretization of $\mathcal{T}_{k}$, which leads to a well-conditioned mixed 


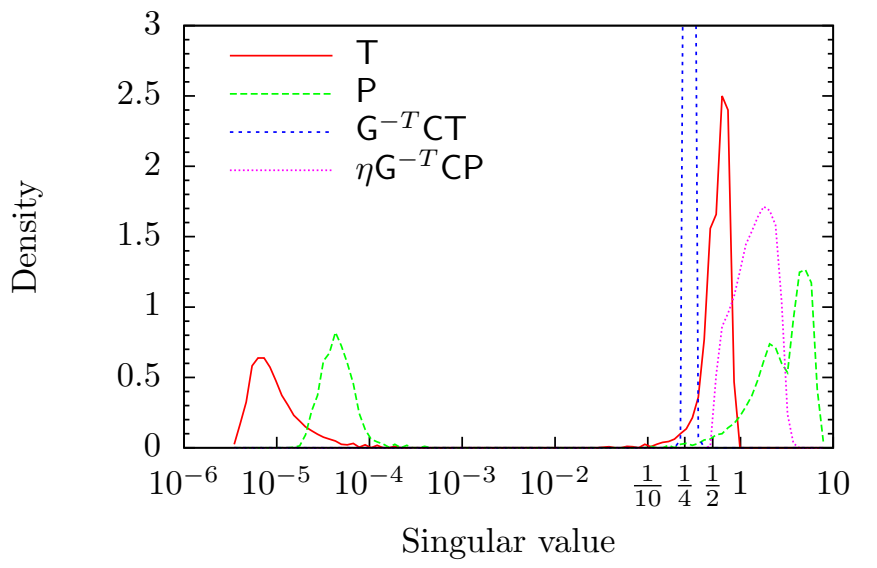

Figure 4: Singular value distribution of $\mathrm{T}$ and $\mathrm{P}$, with and without Calderón preconditioner, for the Luneburg lens with $h / \lambda=1.7 \cdot 10^{-3}$ and $N_{b}=4521$ unknowns.

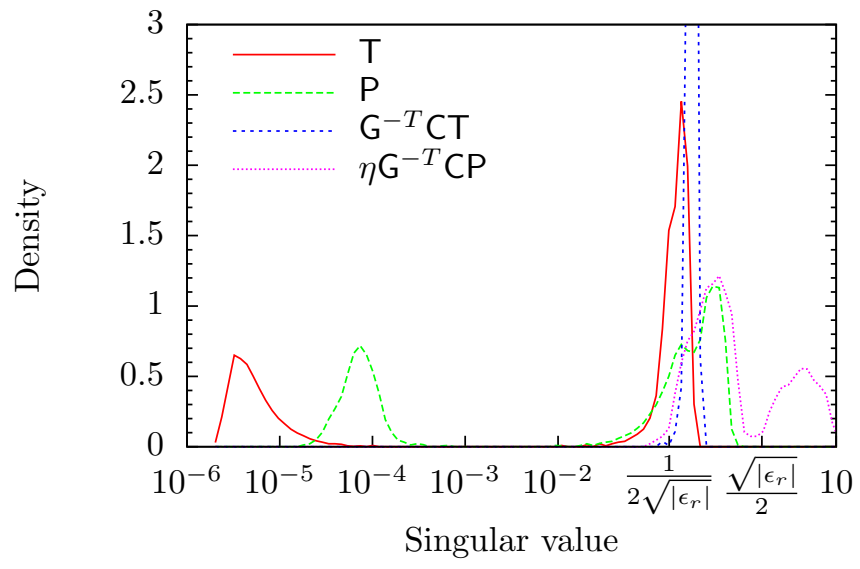

Figure 5: Singular value distribution of $\mathrm{T}$ and $\mathrm{P}$, with and without Calderón preconditioner, for the homogeneous dielectric cube with $\epsilon_{r}=10-0.1 j, h / \lambda=2.3 \cdot 10^{-3}$ and $N_{b}=4962$ unknowns. 
Gram matrix G, ensures that the beneficial spectral properties of $\mathcal{T}_{k} \mathcal{P}$ are inherited by the discretization CP.

Observe in Fig. 3 that the condition number of CP is relatively small and stays constant as a function of the relative mesh size $h / \lambda$. Unlike the diagonal preconditioner, the Calderón multiplicative preconditioner removes the dense mesh discretization breakdown of $P$. This is also reflected by the singular value distributions of the Calderón preconditioned operators $\eta G^{-T} C P$ and $G^{-T} C T$ for the Luneburg lens, shown in Fig. 4. which manifest one cluster of singular values (instead of the two separated branches of $T$ and $P$ ) around $\frac{1}{2}$ and $\frac{1}{4}$, respectively, as expected from Corollary 2.1 and Calderón identity 14. Note that the transposed inverse (well-conditioned) Gram matrix $\mathrm{G}^{-T}$ does not significantly influence the condition number of CT and CP or the behavior of their singular value distribution, but is merely added such that the accumulation points $\frac{1}{2}$ and $\frac{1}{4}$ correspond to those of the continuous operators $\eta \mathcal{T}_{k} \mathcal{P}$ and $\mathcal{T}_{k}^{2}$, respectively.

Fig. 5 illustrates the effect of a discontinuity in the permittivity at the boundary $\Gamma$ on the singular value distribution of the discretized operators, for the example of the homogeneous dielectric cube of $\$ 5.3$ with relative permittivity $\epsilon_{r}=10-0.1 j$. We observe two clusters of singular values of the Calderón preconditioned operator $\eta \mathrm{G}^{-T} \mathrm{CP}$ around $\left|\frac{k^{\prime}}{2 k}\right|=\frac{1}{2}\left|\epsilon_{r}\right|^{-1 / 2}$ and $\left|\frac{k}{2 k^{\prime}}\right|=\frac{1}{2}\left|\epsilon_{r}\right|^{1 / 2}$, in line with Corollary 2.2 (although $\Gamma$ is strictly speaking not smooth), with $k^{\prime}$ the wavenumber of the homogeneous exterior domain in this case, and $k=\sqrt{\epsilon_{r}} k^{\prime}$ and $\eta$ the wavenumber and characteristic impedance of the homogeneous cube, respectively. Therefore, the preconditioner $\mathrm{C}$ (with wavenumber $k^{\prime}$ in the discretization $\mathrm{T}_{\mathrm{bc}}$ ) cures $\mathrm{P}$ (with wavenumber $k$ at the boundary) from dense discretization breakdown, even if $k \neq k^{\prime}$. However, the appearance of two clusters of singular values leads, in general, to a higher condition number of $\eta \mathrm{G}^{-T} \mathrm{CP}$ (whose logarithm is equal to the length of the smallest interval that contains the support of the curves in Figs. 4 and 5 ), in comparison with the case $k=k^{\prime}$.

\section{Preconditioned Reduced Hybrid FEM-BEM Formulation}

The numerical simulation of the scattering problem (1)-(4) at a heterogeneous domain typically combines a boundary element method (BEM) [38, 41, 51, with unknown current densities in a finite-dimensional subspace of $\mathbf{H}^{-\frac{1}{2}}\left(\operatorname{div}_{\Gamma}, \Gamma\right)$, with a finite element method [44, 45, with an unknown electric field in a finitedimensional subspace of $\mathbf{H}\left(\mathrm{curl}, \Omega^{-}\right)$, resulting in a hybrid FEM-BEM method, sometimes called FE-BI (finite element, boundary integral) method [39, 52, 53. In this way, the advantages of both methods, i.e., the natural incorporation of heterogeneous material parameters in FEM and the inherent validity of the radiation condition of the scattered fields in BEM, are combined.

In this section, we propose a hybrid FEM-BEM formulation whose reduced form (after elimination of the interior FEM degrees of freedom) is suitable for Calderón preconditioning. The proposed Calderón multiplicative preconditioner for the reduced FEM-BEM system relies on the fact that C (as introduced in $\$ 3.4$ is a preconditioner for both $\mathrm{T}$ and $\mathrm{P}$.

\subsection{Formulation}

Consider a tangential magnetic current density $\mathbf{m}_{0} \in \mathbf{H}^{-\frac{1}{2}}\left(\operatorname{div}_{\Gamma}, \Gamma\right)$ flowing on $\Gamma$ and generating scattered fields in the exterior domain $\Omega^{+}$(Fig. 1). In order to make the formulation amenable to multiplicative Calderón preconditioning, as explained in the next section, $\mathbf{m}_{0}$ is the only source for the scattered fields in $\Omega^{+}$(no electric current density is assumed). This implies that, in general, $\mathbf{m}_{0}$ is different from the trace of the total tangential electric field, similar to the magnetic current formulation of [54]. Along with the unknown current density $\mathbf{m}_{0}$, we consider this total tangential electric field at the boundary, i.e., the interior limit of $\hat{\mathbf{n}} \times \mathbf{e}$ to the boundary, as another independent unknown of the formulation. The exterior traces of the total tangential electric and magnetic fields follow immediately from (12) and are given by $\hat{\mathbf{n}} \times \mathbf{e}^{(i)}+\left(\mathcal{K}_{k}+\frac{1}{2}\right) \mathbf{m}_{0}$ and $\hat{\mathbf{n}} \times \mathbf{h}^{(i)}+\frac{1}{\eta} \mathcal{T}_{k} \mathbf{m}_{0}$, respectively. The interior traces of the total tangential electric and magnetic fields are given by $\hat{\mathbf{n}} \times \mathbf{e}$ and $\mathcal{P}(\hat{\mathbf{n}} \times \mathbf{e})$, respectively. Expressing the the tangential continuity 
of the electric and magnetic fields at the boundary leads to the following system (41).

$$
\begin{cases}\left(\mathcal{K}_{k}+\frac{1}{2}\right) \mathbf{m}_{0}-\hat{\mathbf{n}} \times \mathbf{e} & =-\hat{\mathbf{n}} \times \mathbf{e}^{(i)} \\ \frac{1}{\eta} \mathcal{T}_{k} \mathbf{m}_{0}-\mathcal{P}(\hat{\mathbf{n}} \times \mathbf{e}) & =-\hat{\mathbf{n}} \times \mathbf{h}^{(i)}\end{cases}
$$

After expanding the unknowns $\mathbf{m}_{0}$ and $\hat{\mathbf{n}} \times \mathbf{e}$ into RWG functions $\hat{\mathbf{n}} \times \mathbf{b}_{b}^{m}$, testing the first equation of 41) with $\hat{\mathbf{n}} \times \mathrm{BC}$ functions $\hat{\mathbf{n}} \times \mathbf{d}^{m}$ and the second equation with $\hat{\mathbf{n}} \times \mathrm{RWG}$ functions $\mathbf{b}_{b}^{m}$, and using equations (35)- $(38)$, we obtain the discretized system

$$
\left(\begin{array}{cc}
\mathrm{K}-\frac{1}{2} \mathrm{G}^{T} & -\mathrm{G}^{T} \\
\mathrm{~T} / \eta & -\mathrm{P}
\end{array}\right)\left(\begin{array}{l}
\bar{\alpha} \\
\bar{\beta}
\end{array}\right)=\left(\begin{array}{l}
\bar{a} \\
\bar{b}
\end{array}\right)
$$

with $\bar{\alpha}$ and $\bar{\beta}$ the expansion coefficients of $\mathbf{m}_{0}$ and $\hat{\mathbf{n}} \times \mathbf{e}$, respectively, $(\bar{a})_{m}=-\int_{\Gamma} \mathbf{d}^{m} \cdot \mathbf{e}^{(i)} \mathrm{d} S$ and $(\bar{b})_{m}=$ $\int_{\Gamma}\left(\hat{\mathbf{n}} \times \mathbf{b}_{b}^{m}\right) \cdot \mathbf{h}^{(i)} \mathrm{d} S$. The presence of the ill-conditioned submatrices $\mathbf{T} / \eta$ and $\mathbf{P}$, which suffer from dense mesh discretization breakdown as explained in Section 3.3 , causes an ill-conditioned system matrix, which negatively affects the convergence speed of an iterative solution algorithm of system (42) (see Section 5).

\subsection{Calderón Multiplicative Preconditioner}

It is well known that the mixed Gram matrix does not suffer from dense mesh discretization breakdown and is well-conditioned [14, 22]. Moreover, the submatrix $\mathrm{K}-\frac{1}{2} \mathrm{G}^{T}$ in $\sqrt{42}$ ) is the so-called mixed discretization of the MFIE (magnetic field integral equation) operator $\mathcal{K}_{k}-\frac{1}{2}$, and is known to be well-conditioned [55]. Taking these observations into account and the fact that $\mathrm{C}$ is a preconditioner for both $\mathrm{T}$ and $\mathrm{P}$, we propose the following Calderón multiplicative preconditioner for hybrid system (42), with I the identity matrix:

$$
\mathrm{C}_{s}=\left(\begin{array}{ll}
\mathrm{I} & 0 \\
0 & \mathrm{C}
\end{array}\right) \text {. }
$$

This leads to the preconditioned reduced hybrid FEM-BEM system

$$
\left(\begin{array}{cc}
\mathrm{K}-\frac{1}{2} \mathrm{G}^{T} & -\mathrm{G}^{T} \\
\mathrm{~T}_{\mathrm{bc}} \mathrm{G}^{-1} \mathrm{~T} / \eta & -\mathrm{T}_{\mathrm{bc}} \mathrm{G}^{-1} \mathrm{P}
\end{array}\right)\left(\begin{array}{c}
\bar{\alpha} \\
\bar{\beta}
\end{array}\right)=\left(\begin{array}{c}
\bar{a} \\
\mathrm{~T}_{\mathrm{bc}} \mathrm{G}^{-1} \bar{b}
\end{array}\right),
$$

Note that the desired property that the preconditioned hybrid system matrix in (44) is well-conditioned does not necessarily follow from the fact that its submatrices are well-conditioned (an illustrative counterexample is a block matrix filled with identity submatrices), but will be shown to hold for representative numerical examples in Section 5. The preconditioner $\mathrm{C}_{s}$ is fully compatible with existing matrix vector product acceleration schemes, such as the multilevel fast multipole algorithm (MLFMA) 31 34.

\subsection{Low-frequency behavior}

Suppose $\Omega^{-}$is simply connected. Then it is well known that the mixed discretization $\mathrm{K}-\frac{1}{2} \mathrm{G}^{T}$ is an accurate and well-conditioned discretization of the MFIE operator $\mathcal{K}_{k}-\frac{1}{2}$ at arbitrarily low frequencies, without the explicit need to refine the mesh [56. Moreover, in absence of numerical cancellation errors in the calculation of $\mathrm{T}, \mathrm{T}_{\mathrm{bc}}$ and the matrix products (which can be circumvented by using a loop-star decomposition), $\mathrm{T}_{\mathrm{bc}} \mathrm{G}^{-1} \mathrm{~T}$ is an accurate and well-conditioned discretization of the Calderón preconditioned EFIE operator $\mathcal{T}_{k}^{2}$ for arbitrarily low frequencies [57. Considering the similarities between the Calderón preconditioned EFIE and PS operators (cf. Corollary 2.1 and Section 3.4), it is expected that $\mathrm{T}_{\mathrm{bc}} \mathrm{G}^{-1} \mathrm{P}$ is an accurate and well-conditioned discretization of the Calderón preconditioned PS operator $\mathcal{T}_{k} \mathcal{P}$ for arbitrarily low frequencies, in which case the preconditioned reduced hybrid FEM-BEM system (44) is expected to be accurate and well-conditioned at arbitrarily low frequencies. However, these statements, along with a loop-star decomposition for $\mathrm{T}_{\mathrm{bc}} \mathrm{G}^{-1} \mathrm{P}$, require further investigation and are beyond the scope of this paper.

For multiply connected $\Omega^{-}$, nontrivial nullspaces of the static limits of the operators $\mathcal{K}_{k}-\frac{1}{2}$ and $\mathcal{T}_{k}^{2}$ lead to ill-conditioning of their respective discretizations $\mathrm{K}-\frac{1}{2} \mathrm{G}^{T}$ and $\mathrm{T}_{\mathrm{bc}} \mathrm{G}^{-1} \mathrm{~T}$ at low frequencies [58]. By Theorem 2.2 the same phenomenon pertains to $\mathcal{T}_{k} \mathcal{P}$, although further investigation is required to solve the resulting ill-conditioning of $T_{b c} G^{-1} P$ at low frequencies for those multiply connected domains. 


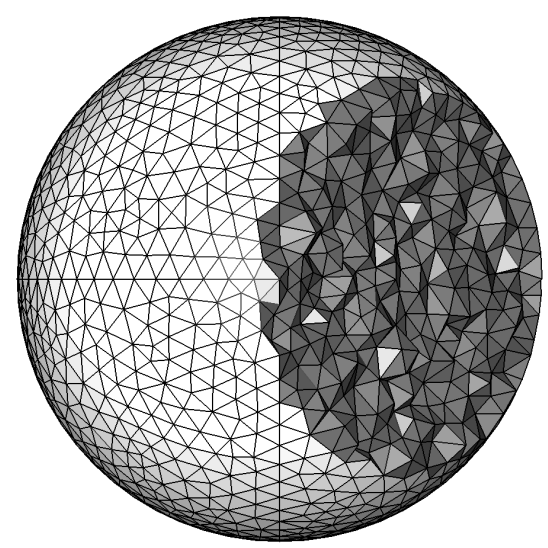

Figure 6: Truncated mesh of a spherical ball with diameter $d$, maximum edge length $h=0.05 d, N_{b}=4491$ boundary edges and $N_{i}=40393$ interior edges.

\section{Numerical Examples}

\subsection{Scattering at a Homogeneous Dielectric Ball}

This example investigates the accuracy of the proposed hybrid formulation (42), for the case of linearly polarized plane wave scattering at a dielectric ball with constant relative permittivity $\epsilon_{r}=\epsilon_{1} / \epsilon_{0}=2$ and diameter $d$. Fig. 7 shows excellent agreement between the scattered electric far field $\lambda^{2} R|\mathbf{e}|$ (scaled with the distance $R$ ), obtained with the hybrid formulation for the mesh of Fig. 6, and the analytical Mie solution [59, 60, for low $(\lambda=10 d$, Rayleigh scattering regime) and high $(\lambda=d)$ frequencies.

\subsection{Scattering at a Luneburg Lens}

In this example the effectiveness of the Calderón multiplicative preconditioner for the hybrid formulation (42) will be investigated. Consider scattering at a classical Luneburg lens, which is a spherical lens with diameter $d$ with spherically symmetric relative permittivity $\epsilon_{r}=\epsilon_{1} / \epsilon_{0}=2-r^{2} /(d / 2)^{2}$ depending on the distance $r$ to its center. In the high frequency limit, i.e. in the ray optics regime (valid for wavelengths $\lambda \ll d)$, it has been proven that a parallel bundle of rays impinging on the lens is focused in a point at the other side on the spherical surface 61. Fig. 8. which results from the solution of the preconditioned hybrid system with the mesh of Fig. 6, shows that this property is still approximately valid for plane wave incidence at $\lambda=0.8 d$.

Figs. 9 and 10 illustrate the effect of dense mesh discretization breakdown of the discretized PoincaréSteklov operator P (as shown in Fig. 3) on the number of iterations and the convergence of the reduced hybrid FEM-BEM system (42), even with diagonal preconditioner. As expected the Calderón multiplicative preconditioner (43), which cures the system from the breakdown, leads to a nearly constant number of iterations for different mesh sizes $h / \lambda$, for fixed wavelength $\lambda$ and precision of the iterative solution algorithm (TFQMR 62] is used in all examples).

The increase in number of iterations is reflected by the execution times in Fig. 11, where it should be noted that the execution time of the TFQMR algorithm with Calderón preconditioner increases for lower $h / \lambda$ because of the increasing dimensionality of the system, but the number of iterations stays constant (see Fig. 9 and Table 1). The calculation of $P$, present in formulation (42), involves the calculation of a Schur complement of the sparse FEM system matrix, as shown in (32). This step is accomplished by the parallel sparse direct multifrontal solver MUMPS [63, and one observes in Fig. 11 that it executes faster than the iterative solution for the considered mesh sizes.

\subsection{Scattering at a Lossy Homogeneous Dielectric Cube}

In the previous example, the boundary $\Gamma$ is smooth, allowing the direct application of Theorem 2.2 and Corollary 2.1 to explain the observed lack of dense mesh discretization breakdown of the preconditioned 


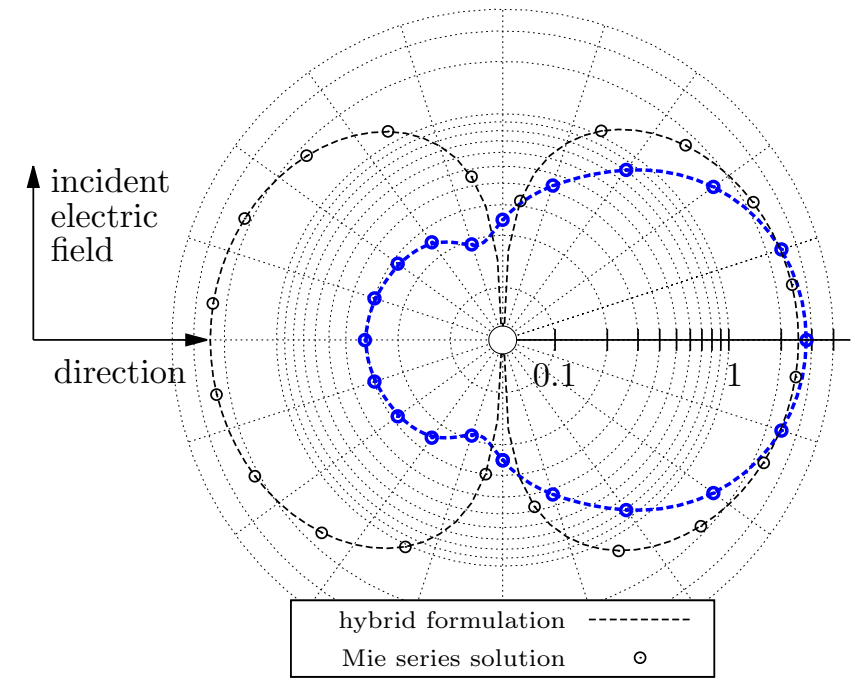

Figure 7: Scattered electric far field for the dielectric ball of Fig. $6\left(\epsilon_{r}=2\right)$ at low $(\lambda=10 d)$ and high $(\lambda=d$, in bold lines and symbols) frequencies.

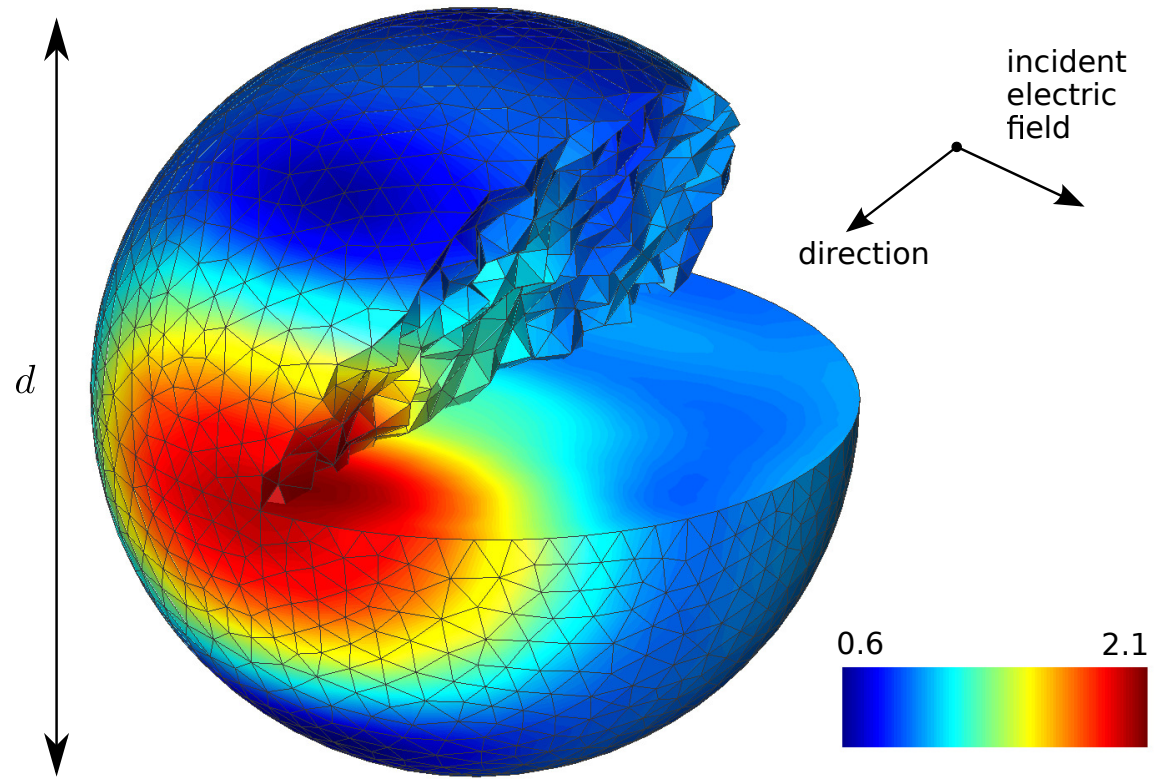

Figure 8: Relative electric field magnitude in a Luneburg lens with diameter $d$ (cut to visualize the interior field) for linearly polarized plane wave scattering with wavelength $\lambda=0.8 d$ and unit electric field magnitude. 


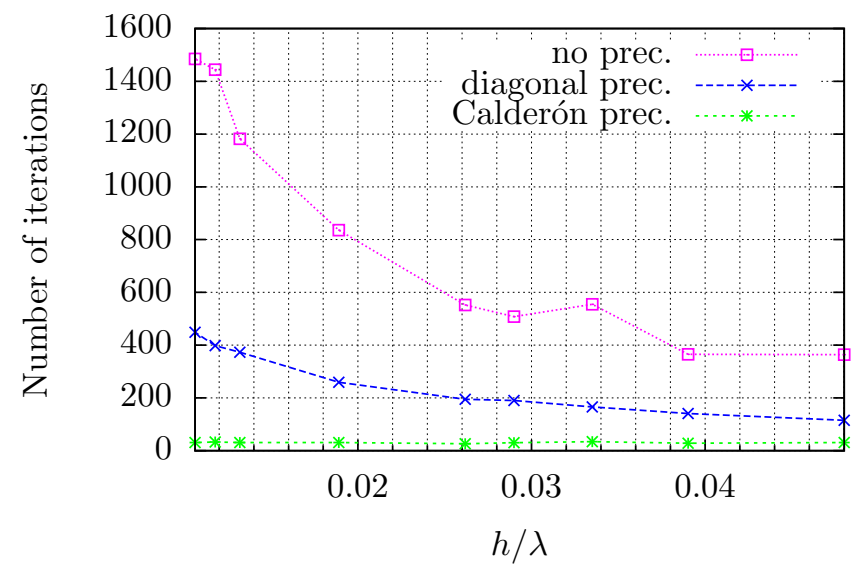

Figure 9: Number of iterations for the solution of 42 (TFQMR, $10^{-9}$ precision) for scattering at the Luneburg lens of Fig. 8 for different preconditioners, as a function of the relative mesh size $h / \lambda($ with $\lambda=4 d$ ).

\begin{tabular}{|c|c|c|c|c|c|}
\hline \multirow{2}{*}{} & $\mathcal{E}$ & \multirow{2}{*}{$h / \lambda$} & \multicolumn{3}{|c|}{ number of iterations } \\
\cline { 4 - 6 } & & & no prec. & diag. prec. & Cald. prec. \\
\hline \multirow{4}{*}{ Luneburg lens } & $10^{-9}$ & $4.8 \cdot 10^{-2}$ & 364 & 115 & 31 \\
\cline { 2 - 6 } & $10^{-9}$ & $2.6 \cdot 10^{-2}$ & 552 & 195 & 26 \\
\cline { 2 - 6 } & $10^{-9}$ & $1.1 \cdot 10^{-2}$ & 1485 & 449 & 31 \\
\cline { 2 - 6 } & $10^{-6}$ & $1.1 \cdot 10^{-2}$ & 1019 & 224 & 22 \\
\cline { 2 - 6 } & $10^{-3}$ & $1.1 \cdot 10^{-2}$ & 406 & 145 & 10 \\
\hline \multirow{4}{*}{ Cube } & $10^{-6}$ & $4.2 \cdot 10^{-2}$ & 148 & 66 & 40 \\
\cline { 2 - 6 } & $10^{-6}$ & $1.0 \cdot 10^{-2}$ & 691 & 215 & 57 \\
\cline { 2 - 6 } & $10^{-6}$ & $4.9 \cdot 10^{-3}$ & 1421 & 395 & 79 \\
\hline \multirow{3}{*}{ Graded-index fiber } & $10^{-6}$ & $5.3 \cdot 10^{-3}$ & 566 & 171 & 29 \\
\cline { 2 - 6 } & $10^{-6}$ & $3.8 \cdot 10^{-3}$ & 950 & 225 & 34 \\
\cline { 2 - 6 } & $10^{-6}$ & $2.0 \cdot 10^{-3}$ & 2223 & 408 & 46 \\
\hline
\end{tabular}

Table 1: Number of iterations for the solution of 422 for scattering at the Luneburg lens (cf. Figs. 9 and 10 , the lossy dielectric cube (cf. Fig. 13 and the bent graded-index fiber (cf. Fig. 15), for different preconditioners, as a function of the relative residual error $\mathcal{E}$ of the iterative solver (TFQMR) and the relative mesh size $h / \lambda$ (with $\lambda=4 d$, $10 d$ and $9 l$, respectively). 


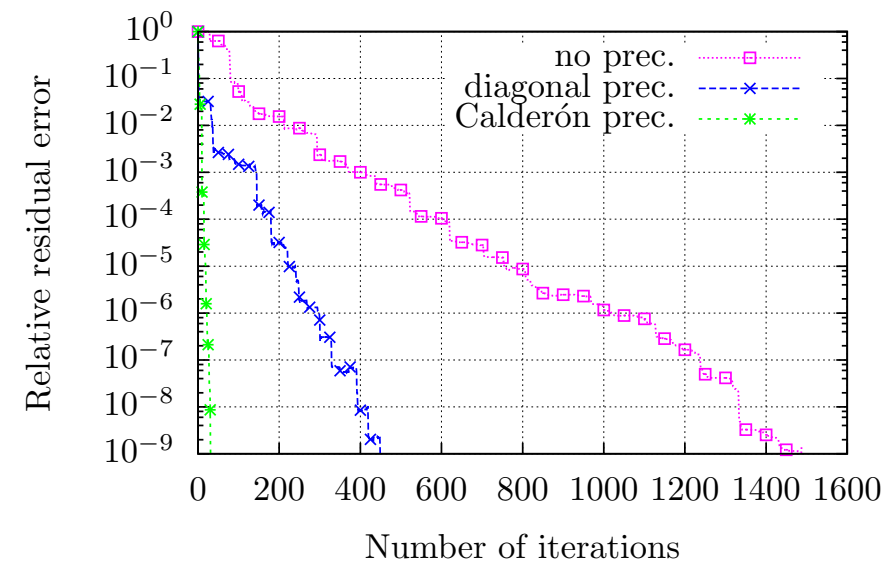

Figure 10: Relative residual error of the iterative solution algorithm (TFQMR) as a function of the number of iterations for the Luneburg lens with $h / \lambda=0.01$ and $2 N_{b}=14016$ total unknowns.

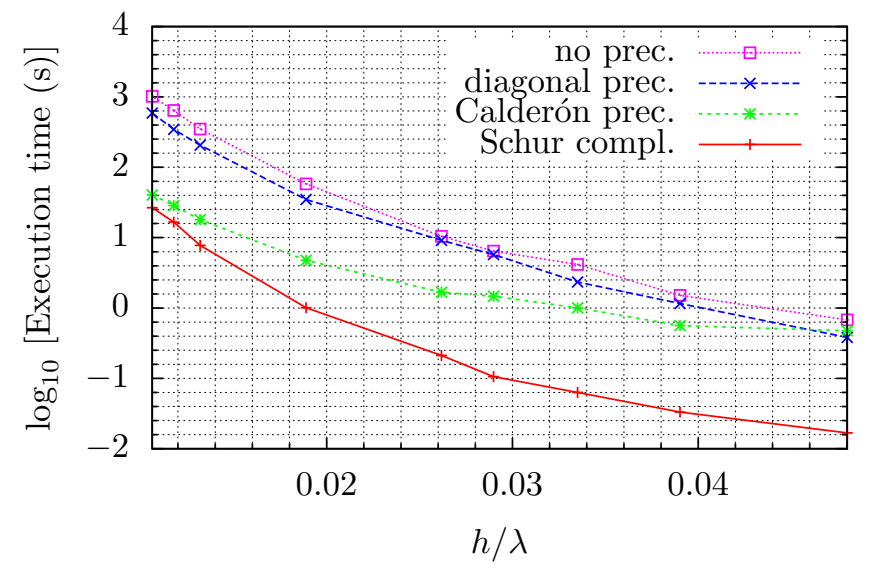

Figure 11: Execution times of the iterative solution for different preconditioners (for the same parameters of Fig. 9) and the Schur complement calculation using MUMPS. 


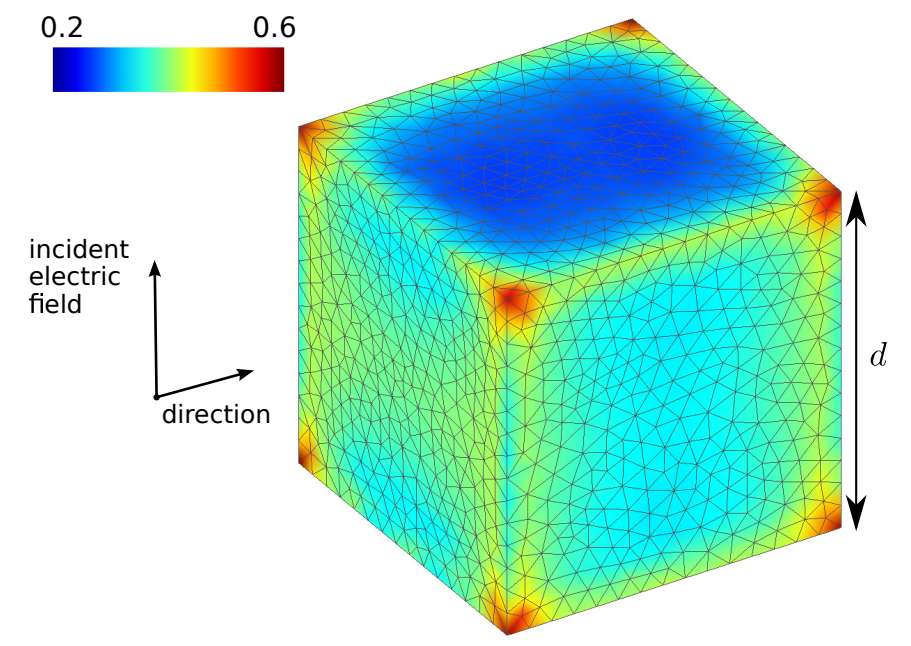

Figure 12: Relative electric field magnitude on the boundary (interior limit) of a lossy dielectric cube with relative permittivity $\epsilon_{r}=10-0.1 j$ and edge length $d$ for linearly polarized plane wave scattering with wavelength $\lambda=10 d$ and unit electric field magnitude.

system (44). In order to investigate the effects of a non-smooth (but Lipschitzian) boundary and a discontinuity of the permittivity at $\Gamma$ (i.e., a configuration where $\lim _{\Omega^{-} \ni \mathbf{r} \rightarrow \Gamma} \epsilon_{1}(\mathbf{r})$ is constant but different from the permittivity $\epsilon_{0}$ of the exterior homogeneous domain $\Omega^{+}$), we consider scattering at the lossy homogeneous dielectric cube of Fig. 12 with relative (with respect to $\epsilon_{0}$ ) permittivity $\epsilon_{r}=10-0.1 j$.

In line with the results of scattering at the Luneburg lens, we observe in Fig. 13 that the Calderón preconditioned system (44) again outperforms the original system (42), with or without diagonal preconditioner, in terms of number of iterations of the iterative solution. However, Table 1 reveals that the number of iterations, for a fixed precision $\mathcal{E}$, still increases slowly for a decreasing relative mesh size $h / \lambda$, which is not the case for the Luneburg lens. Therefore, the slow increase increase in number of iterations can be attributed to the non-smooth boundary, which causes singular tangential electric field components (of the exact solution) around the sharp corners (as can be observed in Fig. 12).

For the same precision $\mathcal{E}=10^{-6}$ and comparable relative mesh sizes $h / \lambda=1.0 \cdot 10^{-2}$ and $h / \lambda=1.1 \cdot 10^{-2}$, we observe in Table 1 that the number of iterations for the cube is significantly higher than for the Luneburg lens (57 versus 22, respectively). This is due the higher condition number of the discrete preconditioned PS operator $\mathrm{T}_{\mathrm{bc}} \mathrm{G}^{-1} \mathrm{P}$ for the cube, which is caused by the jump in permittivity at the boundary, as explained in Section 3.4 and shown in Figs. 4 and 5 (where the logarithm of the condition number is equal to the length of the smallest interval that contains the support of the functions).

\subsection{Scattering at a Bent Graded-index Fiber}

Consider plane wave scattering at a bent graded-index fiber, with parabolically varying permittivity $\epsilon_{1}$ ranging from $4 \epsilon_{0}$ (at the center of the fiber) to $2 \epsilon_{0}$ (on the boundary), as shown in Fig. 14. In addition to its practical relevance, this example is interesting because its permittivity, although in $C^{1}\left(\Omega^{-}\right)$, is not constant on the boundary (it varies parabolically on the two cross sections).

Nevertheless, we observe in Fig. 15 and Table 1 that the Calderón preconditioner (43) cures the system (42) from dense mesh discretization breakdown, as indicated by the nearly constant number of iterations, whereas the number of iterations with diagonal preconditioner increases with decreasing mesh size. This is reflected by the execution times in Fig. 16, where we observe that the relative gain of the Calderón preconditioner w.r.t. the diagonal preconditioner increases for finer meshes, due to the earlier mentioned breakdown occurring with the latter preconditioner. Similar to the results in Fig. 11, the execution time of the iterative solution of the dense reduced hybrid system 42 dominates the calculation of the Schur complement (32) using the direct sparse solver MUMPS, as shown in Fig. 16, for all considered mesh sizes. 


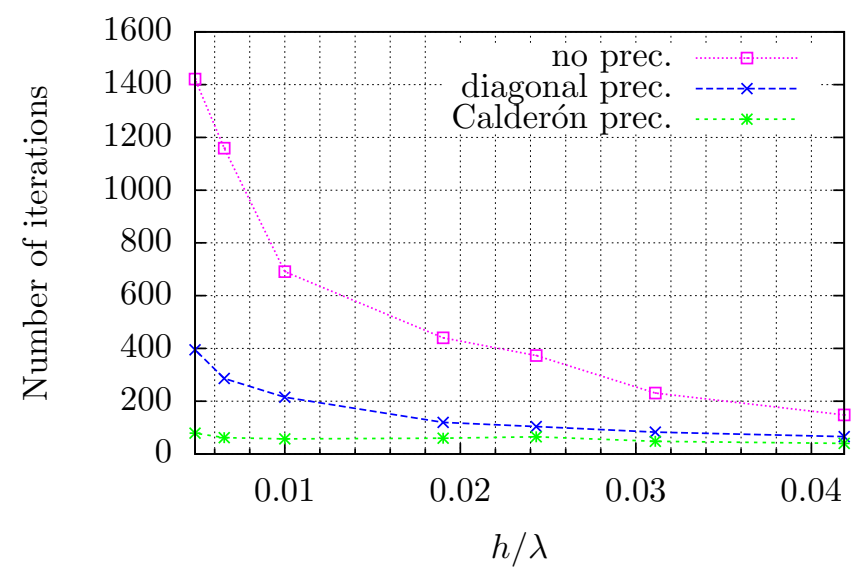

Figure 13: Number of iterations for the solution of 42 (TFQMR, $10^{-6}$ precision) for scattering at the homogeneous dielectric cube of Fig. 12 for different preconditioners, as a function of the relative mesh size $h / \lambda$ (with $\lambda=10 d$ ).



Figure 14: Relative electric field magnitude on the boundary (interior limit) of a bent graded-index fiber (with permittivity varying parabolically from $4 \epsilon_{0}$ at the center to $2 \epsilon_{0}$ on the boundary) with length $l$ and diameter $d$ for linearly polarized plane wave scattering with wavelength $\lambda=5 d=0.9 l$ and unit electric field magnitude.

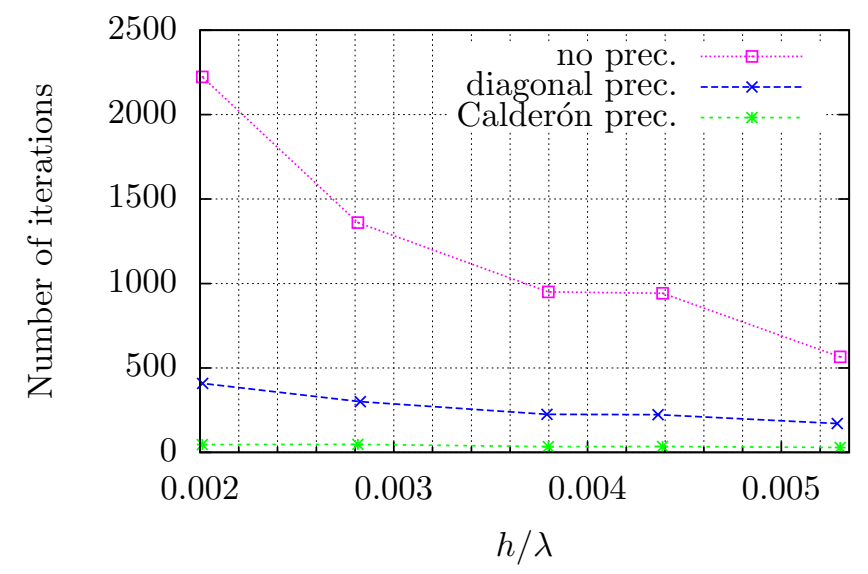

Figure 15: Number of iterations for the solution of 42 (TFQMR, $10^{-6}$ precision) for scattering at the bent graded-index fiber of Fig. 14 for different preconditioners, as a function of the relative mesh size $h / \lambda$ (with $\lambda=9 l$ ). 


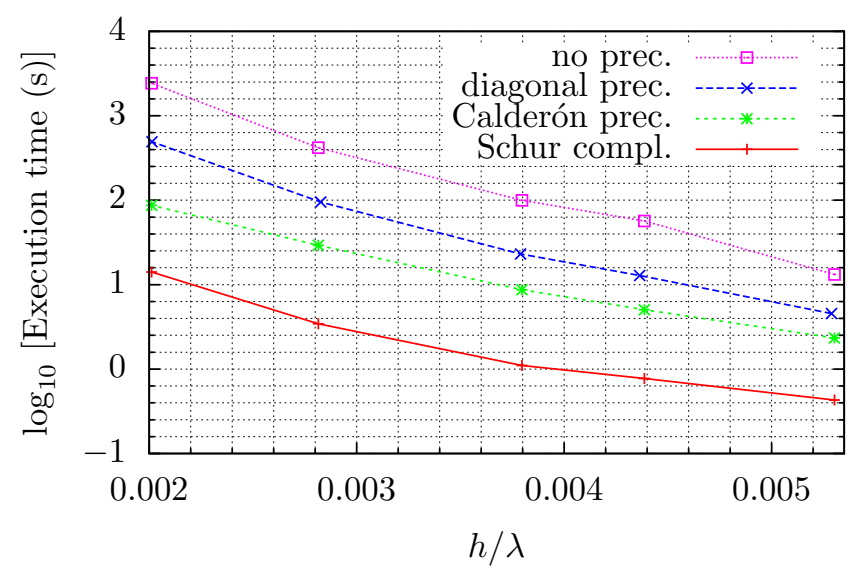

Figure 16: Execution times of the iterative solution for different preconditioners (for the same parameters of Fig. 15 and the Schur complement calculation using MUMPS.

\section{Acknowledgements}

The first author is supported by a doctoral grant from the Research Foundation Flanders (FWO-V). The authors gratefully acknowledge the anonymous reviewers for their valuable comments and suggestions that helped to improve the paper significantly. The authors made extensive use of freely distributed software including libMesh 64, MUMPS 63] and Gmsh 65].

\section{Conclusions}

We prove that the electric field boundary integral operator can be used to regularize the continuous timeharmonic electromagnetic Poincaré-Steklov operator of a heterogeneous domain. The beneficial implications of a discrete Calderón multiplicative preconditioner (CMP) on the condition number of its finite element discretization are numerically investigated. This paves the way for an efficient iterative solution of reduced hybrid FEM-BEM problems. We demonstrate the accuracy of a formulation that is amenable to the CMP on a canonical problem and the effectiveness of the preconditioner in practically relevant scattering problems.

\section{References}

[1] R. R. Paz, N. M. Nigro, M. A. Storti, On the efficiency and quality of numerical solutions in CFD problems using the interface strip preconditioner for domain decomposition methods, International journal for numerical methods in fluids 52 (1) (2006) 89-118.

[2] P. Demarcke, H. Rogier, The Poincaré-Steklov operator in hybrid finite element-boundary integral equation formulations, IEEE Antennas and Wireless Propagation Letters 10 (2011) 503-506. doi:10.1109/LAWP.2011.2157072

[3] F. Boeykens, H. Rogier, J. Van Hese, J. Sercu, T. Boonen, Rigorous analysis of internal resonances in 3-D hybrid FE-BIE formulations by means of the Poincaré-Steklov operator, Microwave Theory and Techniques, IEEE Transactions on 61 (10) (2013) 3503-3513. doi:10.1109/TMTT.2013.2277990

[4] L. F. Knockaert, D. D. Zutter, On the complex symmetry of the Poincaré-Steklov operator, Progress In Electromagnetics Research B 7 (2008) 145-157. doi:10.2528/PIERB08022102.

[5] F. Alouges, S. Borel, D. Levadoux, A stable well-conditioned integral equation for electromagnetism scattering Journal of Computational and Applied Mathematics 204 (2) (2007) 440 - 451, special Issue: The Seventh International Conference on Mathematical and Numerical Aspects of Waves (WAVES'05). doi:http://dx.doi.org/10.1016/j.cam.2006.02.049 URL http://www.sciencedirect.com/science/article/pii/S0377042706003712

[6] A. Quarteroni, A. Valli, Theory and application of Steklov-Poincaré operators for boundary-value problems in: R. Spigler (Ed.), Applied and Industrial Mathematics, Vol. 56 of Mathematics and Its Applications, Springer Netherlands, 1991, pp. 179-203. doi:10.1007/978-94-009-1908-2_14

URL http://dx .doi .org/10.1007/978-94-009-1908-2_14 
[7] V. I. Agoshkov, Poincaré-Steklov operators and domain decomposition methods in finite dimensional spaces, in: R. Glowinski, G. Golub, G. Meurant, J. Périaux (Eds.), First international symposium on domain decomposition methods for partial differential equations, SIAM, 1988, p. 73112.

[8] A. Toselli, O. Widlund, Domain Decomposition Methods - Algorithms and Theory, Springer Series in Computational Mathematics, Springer, 2005.

[9] A. Krebs, M. Maischak, E. P. Stephan, Adaptive FEM-BEM coupling with a Schur complement error indicator., Appl. Numer. Math. 60 (8) (2010) 798-808. doi:10.1016/j.apnum.2010.04.004

[10] G. C. Hsiao, W. L. Wendland, Boundary integral equations, Applied Mathematical Sciences, Springer, Berlin, Heidelberg, 2008.

[11] M. A. Storti, L. Dalcín, R. Paz, A. Yommi, V. Sonzogni, N. Nigro, A preconditioner for the Schur complement matrix., Advances in Engineering Software 37 (11) (2006) 754-762.

[12] A. Gillman, A. Barnett, P.-G. Martinsson, A spectrally accurate direct solution technique for frequency-domain scattering problems with variable media, BIT Numerical Mathematics 55 (1) (2015) 141-170. doi:10.1007/s10543-014-0499-8 URL http://dx.doi.org/10.1007/s10543-014-0499-8

[13] R. Adams, Physical and analytical properties of a stabilized electric field integral equation, Antennas and Propagation, IEEE Transactions on 52 (2) (2004) 362-372.

[14] F. Andriulli, K. Cools, H. Bagci, F. Olyslager, A. Buffa, S. Christiansen, E. Michielssen, A multiplicative Calderon preconditioner for the electric field integral equation, Antennas and Propagation, IEEE Transactions on 56 (8) (2008) 2398-2412. doi:10.1109/TAP.2008.926788

[15] S. Adrian, F. Andriulli, T. Eibert, A Calderón preconditioner for the EFIE operator without barycentric refinement of the mesh, in: Antennas and Propagation Society International Symposium (APSURSI), 2014 IEEE, 2014 , pp. $2180-2181$. doi:10.1109/APS.2014.6905417

[16] H. Bagci, F. P. Andriulli, K. Cools, F. Olyslager, E. Michielssen, A Calderón multiplicative preconditioner for the combined field integral equation, Antennas and Propagation, IEEE Transactions on 57 (10) (2009) 3387-3392.

[17] Y. Boubendir, C. Turc, Well-conditioned boundary integral equation formulations for the solution of high-frequency electromagnetic scattering problems Computers and Mathematics with Applications 67 (10) (2014) $1772-1805$. doi: http://dx.doi.org/10.1016/j.camwa.2014.04.003 URL http://www.sciencedirect.com/science/article/pii/S0898122114001564

[18] F. Valdes, F. Andriulli, H. Bagci, E. Michielssen, A Calderón-preconditioned single source combined field integral equation for analyzing scattering from homogeneous penetrable objects, Antennas and Propagation, IEEE Transactions on 59 (6) (2011) 2315-2328. doi:10.1109/TAP.2011.2143671

[19] K. Cools, F. Andriulli, E. Michielssen, A Calderón multiplicative preconditioner for the PMCHWT integral equation, Antennas and Propagation, IEEE Transactions on 59 (12) (2011) 4579-4587. doi:10.1109/TAP.2011.2165465.

[20] P. Yla-Oijala, S. Kiminki, S. Jarvenpaa, Calderon preconditioned surface integral equations for composite objects with junctions, Antennas and Propagation, IEEE Transactions on 59 (2) (2011) 546-554. doi:10.1109/TAP.2010.2096192

[21] Y. Chen, S. Sun, L. Jiang, W. C. Chew, A Calderón preconditioner for the electric field integral equation with layered medium Green's function, Antennas and Propagation, IEEE Transactions on 62 (4) (2014) 2022-2030. doi:10.1109/TAP. 2013.2297396

[22] A. Buffa, S. H. Christiansen, A dual finite element complex on the barycentric refinement, Comptes Rendus Mathematique 340 (6) (2005) 461-464. doi:http://dx.doi.org/10.1016/j.crma.2004.12.022

[23] S. Rao, D. Wilton, A. Glisson, Electromagnetic scattering by surfaces of arbitrary shape, Antennas and Propagation, IEEE Transactions on 30 (3) (1982) 409-418. doi:10.1109/TAP.1982.1142818

[24] J. Liu, J.-M. Jin, A highly effective preconditioner for solving the finite element-boundary integral matrix equation of 3-D scattering, Antennas and Propagation, IEEE Transactions on 50 (9) (2002) 1212-1221. doi:10.1109/TAP.2002.801377

[25] J. Lee, C. Zhang, C.-C. Lu, Sparse inverse preconditioning of multilevel fast multipole algorithm for hybrid integral equations in electromagnetics, Antennas and Propagation, IEEE Transactions on 52 (9) (2004) 2277-2287. doi:10.1109/ TAP. 2004.834084

[26] M.-L. Yang, X.-Q. Sheng, Hybrid h- and p-type multiplicative Schwarz (h-p-MUS) preconditioned algorithm of higherorder FE-BI-MLFMA for 3D scattering, Magnetics, IEEE Transactions on 48 (2) (2012) 187-190. doi:10.1109/TMAG. 2011.2174033

[27] L. Li, T.-Z. Huang, G.-H. Cheng, Y.-F. Jing, Z.-G. Ren, H.-B. Li, Solution to 3-D electromagnetic problems discretized by a hybrid FEM/MOM method, Computer Physics Communications 184 (1) (2013) 73 - 78 . doi:http://dx.doi.org/ 10.1016/j.cpc.2012.08.017 URL http://www.sciencedirect.com/science/article/pii/S0010465512002834

[28] O. Wiedenmann, L. Li, T. Eibert, A direct hierarchical multilevel preconditioner for the solution of finite element-boundary integral equations, in: Antennas and Propagation (EuCAP), 2014 8th European Conference on, 2014 , pp. 3412-3416. doi:10.1109/EuCAP.2014.6902562

[29] Y. Boubendir, A. Bendali, M. B. Fares, Coupling of a non-overlapping domain decomposition method for a nodal finite element method with a boundary element method, International Journal for Numerical Methods in Engineering 73 (11) (2008) 1624-1650. doi:10.1002/nme.2136. URL http://dx.doi.org/10.1002/nme.2136

[30] M.-L. Yang, H.-W. Gao, W. Song, X.-Q. Sheng, An effective domain-decomposition-based preconditioner for the FE-BIMLFMA method for 3D scattering problems, Antennas and Propagation, IEEE Transactions on 62 (4) (2014) $2263-2268$. doi:10.1109/TAP.2014.2300159

[31] L. Greengard, V. Rokhlin, A fast algorithm for particle simulations, J. Comput. Phys. 73 (2) (1987) 325-348. doi: 
10.1016/0021-9991(87)90140-9

URL http://dx.doi.org/10.1016/0021-9991(87)90140-9

[32] V. Rokhlin, Rapid solution of integral equations of scattering theory in two dimensions J. Comput. Phys. 86 (2) (1990) 414-439. doi:10.1016/0021-9991(90)90107-C

URL http://dx.doi.org/10.1016/0021-9991(90)90107-C

[33] W. Chew, E. Michielssen, J. M. Song, J. M. Jin (Eds.), Fast and Efficient Algorithms in Computational Electromagnetics, Artech House, Inc., Norwood, MA, USA, 2001.

[34] H. Cheng, W. Y. Crutchfield, Z. Gimbutas, L. F. Greengard, J. F. Ethridge, J. Huang, V. Rokhlin, N. Yarvin, J. Zhao, A wideband fast multipole method for the helmholtz equation in three dimensions, Journal of Computational Physics 216 (1) (2006) 300-325.

[35] F. Andriulli, K. Cools, I. Bogaert, E. Michielssen, On a Well-Conditioned Electric Field Integral Operator for Multiply Connected Geometries, IEEE transactions on antennas and propagation 61 (4) (2013) $2077-2087$.

[36] J. Van Bladel, Electromagnetic Fields, IEEE Press Series on Electromagnetic Wave Theory, John Wiley \& Sons, 2007.

[37] J. Nédélec, Acoustic and Electromagnetic Equations: Integral Representations for Harmonic Problems, Springer, 2001.

[38] S. Sauter, C. Schwab, Boundary Element Methods, Springer Series in Computational Mathematics, Springer, 2010.

[39] R. Hiptmair, Coupling of finite elements and boundary elements in electromagnetic scattering, SIAM Journal on Numerical Analysis 41 (3) (2004) 919-944.

[40] R. A. Adams, J. J. Fournier, Sobolev Spaces, Pure and Applied Mathematics, Elsevier, 2003.

[41] A. Buffa, R. Hiptmair, Galerkin boundary element methods for electromagnetic scattering, in: M. Ainsworth, P. Davies, D. Duncan, B. Rynne, P. Martin (Eds.), Topics in Computational Wave Propagation, Vol. 31 of Lecture Notes in Computational Science and Engineering, Springer Berlin Heidelberg, 2003, pp. 83-124. doi:10.1007/978-3-642-55483-4_3

[42] G. Hanson, A. Yakovlev, Operator Theory for Electromagnetics: An Introduction, Springer, 2002.

[43] D. Colton, R. Kress, Inverse Acoustic and Electromagnetic Scattering Theory, Applied Mathematical Sciences, Springer, 1998.

[44] P. Monk, Finite Element Methods for Maxwell's Equations, Numerical Mathematics and Scientific Computation, Clarendon Press, 2003.

[45] J. Jin, The Finite Element Method in Electromagnetics, 3rd Edition, Wiley-IEEE Press, 2014.

[46] J. Nédélec, Mixed finite elements in $\mathbb{R}_{3}$, Numerische Mathematik 35 (3) (1980) 315-341. doi:10.1007/BF01396415

[47] L. N. Trefethen, D. Bau, Numerical Linear Algebra, SIAM, 1997.

[48] F. Andriulli, A. Tabacco, G. Vecchi, Solving the EFIE at low-frequencies with a conditioning that grows only logarithmically with the number of unknowns, IEEE transactions on antennas and propagation 58 (05) (2010) $1614-1624$.

[49] S. Yan, J.-M. Jin, Z. Nie, Calderón preconditioner: From EFIE and MFIE to N-Müller equations, Antennas and Propagation, IEEE Transactions on 58 (12) (2010) 4105-4110. doi:10.1109/TAP.2010.2078460

[50] J. Zhu, Y. Hu, R. Chen, H. Zhu, Calderon multiplicative preconditioner based on curvilinear elements for fast analysis of electromagnetic scattering, Microwaves, Antennas Propagation, IET 5 (1) (2011) 102-112. doi:10.1049/iet-map.2009. 0500 .

[51] R. F. Harrington, Field Computation by Moment Methods, Wiley-IEEE Press, 1993.

[52] T. Eibert, V. Hansen, Calculation of unbounded field problems in free space by a 3D FEM/BEM-hybrid approach, Journal of Electromagnetic Waves and Applications 10 (1) (1996) 61-78. doi:10.1163/156939396X00216

[53] J. Volakis, K. Sertel, B. Usner, Frequency Domain Hybrid Finite Element Methods for Electromagnetics, Synthesis Lectures on Computational Electromagnetics, Morgan \& Claypool Publishers, 2006.

[54] R. F. Harrington, Boundary integral formulations for homogeneous material bodies, Journal of Electromagnetic Waves and Applications 3 (1) (1989) 1-15. doi:10.1163/156939389X00016

[55] K. Cools, F. Andriulli, D. De Zutter, E. Michielssen, Accurate and conforming mixed discretization of the MFIE , IEEE antennas and wireless propagation letters 10 (2011) $528-531$.

[56] I. Bogaert, K. Cools, F. Andriulli, H. Bagci, Low-frequency scaling of the standard and mixed magnetic field and Müller integral equations, Antennas and Propagation, IEEE Transactions on 62 (2) (2014) 822-831. doi:10.1109/TAP.2013. 2293783

[57] S. Yan, J.-M. Jin, Z. Nie, EFIE analysis of low-frequency problems with loop-star decomposition and Calderón multiplicative preconditioner, Antennas and Propagation, IEEE Transactions on 58 (3) (2010) 857-867. doi:10.1109/TAP.2009. 2039336

[58] K. Cools, F. Andriulli, F. Olyslager, E. Michielssen, Nullspaces of MFIE and Calderón preconditioned EFIE operators applied to toroidal surfaces, Antennas and Propagation, IEEE Transactions on 57 (10) (2009) 3205-3215. doi:10.1109/ TAP. 2009.2028669.

[59] G. Mie, Beiträge zur Optik trüber Medien, speziell kolloidaler Metallösungen, Annalen der Physik 330 (1908) $377-445$. doi:10.1002/andp.19083300302

[60] J. A. Stratton, Electromagnetic Theory, 1st Edition, McGraw-Hill Companies (New York), 1941.

[61] R. K. Luneburg, Mathematical Theory of Optics, Brown U. Press, Providence, R.I., 1944.

[62] R. W. Freund, A transpose-free quasi-minimal residual algorithm for non-Hermitian linear systems, SIAM J. Sci. Comput. 14 (2) (1993) 470-482. doi:10.1137/0914029

[63] P. R. Amestoy, I. S. Duff, J.-Y. L'Excellent, J. Koster, A fully asynchronous multifrontal solver using distributed dynamic scheduling, SIAM J. Matrix Anal. Appl. 23 (1) (2001) 15-41. doi:10.1137/S0895479899358194.

[64] B. S. Kirk, J. W. Peterson, R. H. Stogner, G. F. Carey, libMesh: A C++ Library for Parallel Adaptive Mesh Refinement/Coarsening Simulations, Engineering with Computers 22 (3-4) (2006) 237-254.

[65] C. Geuzaine, J.-F. Remacle, Gmsh: A 3-D finite element mesh generator with built-in pre-and post-processing facilities, 
International Journal for Numerical Methods in Engineering 79 (11) (2009) 1309-1331. 